\title{
Genomic diversity and biosynthetic capabilities of sponge-associated chlamydiae
}

Jennah E. Dharamshi ${ }^{1,}$, Natalia Gaarslev ${ }^{1}$, Karin Steffen ${ }^{2}$, Tom Martin ${ }^{1}$, Detmer Sipkema ${ }^{3}$, Thijs J. G. Ettema ${ }^{3, *}$

${ }^{1}$ Department of Cell and Molecular Biology, Science for Life Laboratory, Uppsala University, SE-75123 Uppsala, Sweden

${ }^{2}$ Department of Pharmaceutical Biosciences, Biomedical Center, Uppsala University, SE-75123 Uppsala, Sweden

${ }^{3}$ Laboratory of Microbiology, Wageningen University, 6708 WE Wageningen, The Netherlands

*Corresponding authors, thijs.ettema@wur.nl and jennah.dharamshi@gmail.com 


\section{ABSTRACT}

1 Sponge microbiomes contribute to host health, nutrition, and defense through the production of 2 secondary metabolites. Chlamydiae, a phylum of obligate intracellular bacteria ranging from 3 animal pathogens to endosymbionts of microbial eukaryotes, are frequently found associated with

4 sponges. However, sponge-associated chlamydial diversity has not yet been investigated at the 5 genomic level and host-interactions remain thus far unexplored. Here, we sequenced the

6 microbiomes of three sponge species and found high, though variable, Chlamydiae relative 7 abundances of up to $21.2 \%$ of bacterial diversity. Using genome-resolved metagenomics 18 high8 quality sponge-associated chlamydial genomes were reconstructed, covering four chlamydial 9 families. Among these, Sorochlamydiaceae shares a common ancestor with Chlamydiaceae animal

10 pathogens, suggesting long-term co-evolution with animals. Sponge-associated chlamydiae 11 genomes mostly resembled environmental chlamydial endosymbionts, but not pathogens, and 12 encoded genes for degrading diverse compounds associated with sponges, such as taurine. 13 Unexpectedly, we identified widespread genetic potential for secondary metabolite biosynthesis 14 across Chlamydiae, which may represent an explored reservoir of novel natural products. This 15 finding suggests that chlamydiae may partake in defensive symbioses and that secondary 16 metabolites play a wider role in mediating intracellular interactions. Furthermore, sponge17 associated chlamydiae relatives were found in other marine invertebrates, pointing towards wider 18 impacts of this phylum on marine ecosystems.

\section{INTRODUCTION}

19 Porifera (i.e., sponges) are ubiquitous filter-feeding metazoans that provide essential ecosystem 20 services. These animals have complex, deeply integrated, and essential microbiomes that play 21 important roles, such as in global nutrient cycling (1-4). The sponge microbiome also produces 22 secondary (or specialized) metabolites that may contribute to host chemical defence (5). Generally, 23 sponges are known as a source of novel secondary metabolites with medical and industrial 24 relevance (6-9). With increasing exposure to anthropogenic threats, further investigation of the 25 sponge microbiome is essential for understanding host impacts, from acting as detrimental 26 opportunists to providing resilience against dysbiosis and disease $(4,10)$. Many sponge-associated 27 microbial groups remain uncultured to date and have only recently been explored through 28 cultivation-independent sequencing approaches $(11,12)$. In a recent large-scale survey, sponge- 
associated microbial communities were shown to be diverse, yet structured, and composed of taxonomic groups with both generalist and specialist host ranges (13). One of these generalist phyla

31 is Chlamydiae, which is found at high relative abundance in some sponge species $(14,15)$. Yet, the

32 genomic diversity of sponge-associated chlamydiae has not been previously investigated.

33 Chlamydiae is a bacterial phylum of obligate eukaryotic endosymbionts well-known for animal 34 pathogens, such as Chlamydia trachomatis and other Chlamydiaceae (16-18). Though many 35 chlamydiae instead infect microbial eukaryotes and have more extensive metabolic repertoires (16, 36 18, 19). Chlamydial environmental distribution and abundance has been underestimated, as their 37 16S rRNA genes are often missed by primers used to survey microbial diversity (20-22). However, 38 with the recent use of cultivation-independent approaches sequenced chlamydial genomic diversity 39 is quickly expanding, resulting in a widening view of the potential lifestyles of uncultivated 40 chlamydial groups (22-27). Retrieving additional chlamydiae genomes is needed to further our 41 understanding of their ecological impacts, range of host interactions along the parasite-mutualist 42 spectrum, and the evolution of endosymbiosis and pathogenicity $(28,29)$.

43 The sponge species Halichondria panicea, Haliclona oculata, and Haliclona xena, sampled 44 from an estuary in The Netherlands, were previously found to have high chlamydiae relative 45 abundances (15). In the present study, we performed genome-centered analyses of these 46 Chlamydiae in order to gain insight into their sponge-associated lifestyle. Comparative analyses of

4718 high-quality sponge-associated Chlamydiae draft genomes revealed degradative capacity also 48 found in other sponge symbionts, and that they share metabolic features with environmental 49 chlamydiae lineages but that are typically absent from known animal pathogens. Unexpectedly, we 50 also identified extensive genetic potential for secondary metabolite biosynthesis across the 51 Chlamydiae phylum. Finally, we found that relatives of these sponge-associated chlamydiae are 52 also associated with additional sponge species and other marine invertebrates, indicating that 53 chlamydiae have important ecological impacts on animals found in marine ecosystems and 54 represent an untapped reservoir for secondary metabolite discovery.

\section{RESULTS AND DISCUSSION}

\section{Specific chlamydiae lineages vary in relative abundance across three sponge species}

55 Using bacterial-specific small-subunit (SSU) rRNA gene amplicon sequencing high relative 56 abundances of Chlamydiae were found in the sponges $H$. panicea $\mathrm{P} \_\mathrm{S} 1(3.9 \%)$, H. panicea $\mathrm{P} \_\mathrm{S} 2$ 
57 (2.8\%), and H. oculata $\mathrm{O} \_\mathrm{S} 4(21.2 \%)$ (Figure 1a), which had been collected during the same sampling event as a prior study (15) (Figure S1 and Data S1). However, Chlamydiae relative abundance was substantially lower in three additional sponge specimens, H. panicea P_S3 (0.51 \%), H. oculata O_S5 (0.21\%), and H. xena X_S6 (0.26\%) (Figure 1a), that were collected from a similar location, but at different dates (Figure S1 and Data S1). This variation was surprising, as Chlamydiae were previously found to have consistently high relative abundance across all three sponge species (15). Although, Chlamydiae were not detected in other studies of the H. panicea microbiome (30). Beyond Chlamydiae, bacterial composition across the sponge species was consistent with a prior investigation, with the same phyla represented and Proteobacteria being the most dominant phylum (Figure 1a) (15). SSU rRNA gene amplicon sequences were subsequently clustered into operational-taxonomic-units (OTUs) at species-level (97\% identity) (Data S2), revealing variability in the specific bacterial OTUs present in different sponge specimens (Figure 1b). However, three specific chlamydiae OTUs with high relative abundances (up to 8\%; Data S3) were found in all samples with higher overall abundances of Chlamydiae (P_S1, P_S2, and O_S4) (Figure 1a-b). Yet, these OTUs were largely undetected in amplicon data from the other sponge specimens (P_S3, O_S5, and X_S6) (Data S3). In a prior study, these same chlamydial lineages were found at high relative abundance across all specimens of the three sponge species, but were largely undetected in surrounding seawater (15). Together, these results support a close association of these specific chlamydial lineages with the investigated sponge species, while suggesting that any association or increase in abundance is transient.

Chlamydiae have also been detected with varying presence and abundance across a diverse range of other sponge species (13). Despite this, sponge-associated chlamydiae have not been previously investigated at the genome level. With the aim of exploring sponge-associated chlamydial genomic diversity, we sequenced and assembled seven high-quality metagenomes from the six sampled sponge specimens, with two generated for O_S4 (O_S4.1 and O_S4.2) (Figure S1 and Data S1). We assessed microbial diversity in the resulting metagenomes by identifying contigs encoding ribosomal protein gene clusters, and thus representing distinct microbial lineages. Ribosomal protein sequences from each contig were concatenated and a maximum-likelihood (ML) phylogenetic tree reconstructed (Figure 1c and Data S4-S5). Relative abundance of each microbial lineage was inferred by comparing coverage of these contigs from each sponge metagenome (Figure 1c and Data S4). These analyses confirmed the larger patterns in sponge 
a

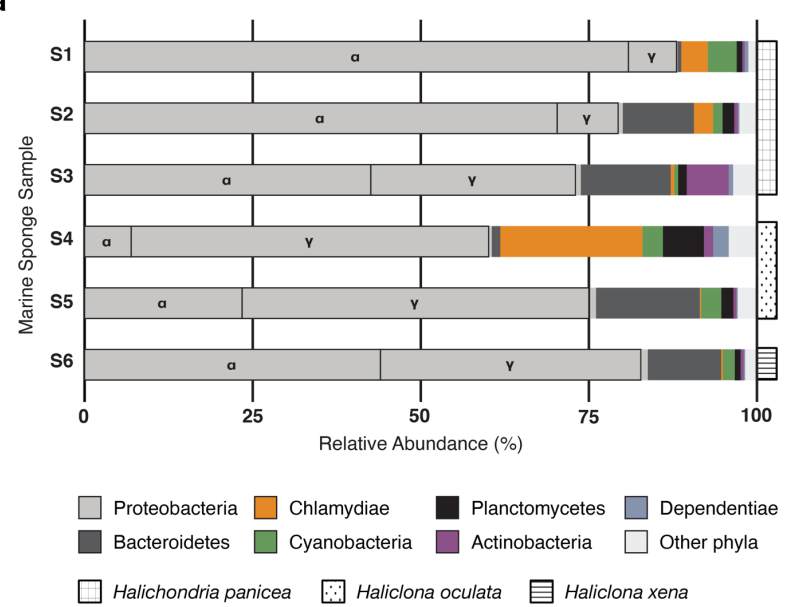

b

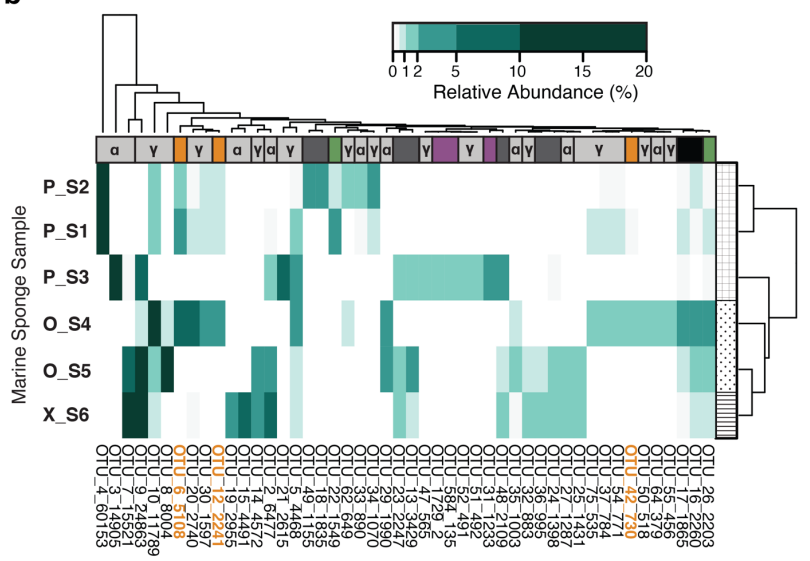

c

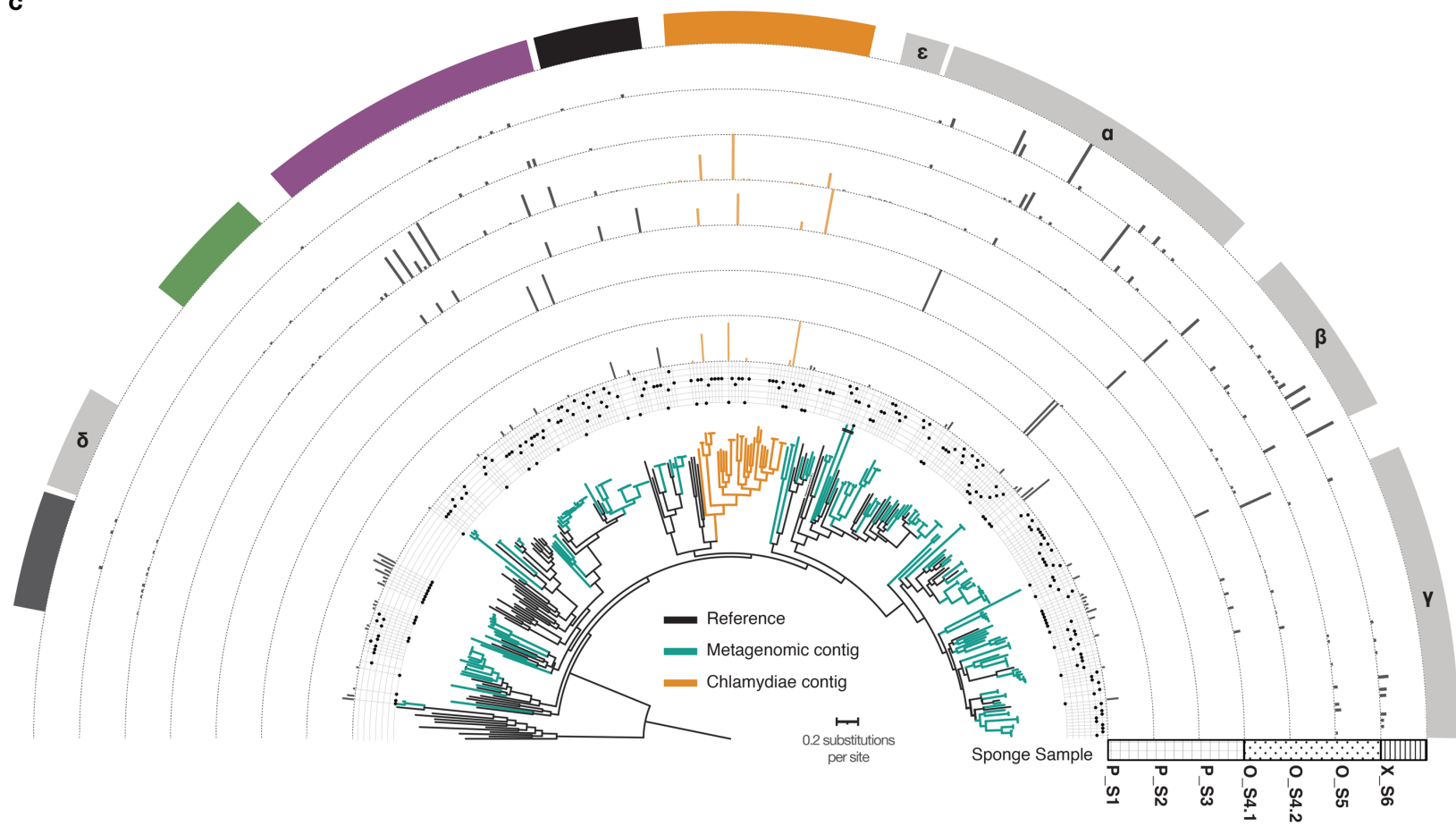

Figure 1. Bacterial SSU rRNA gene amplicon sequencing and metagenomes revealed variably high relative abundances of Chlamydiae across sponge specimens. a. Relative abundances of phyla with $\geq 1 \%$ relative abundance across samples. b. Abundance heat map of OTUs with $\geq 1 \%$ relative abundance in a sponge sample. Both OTUs and sponges are hierarchically clustered based on presence patterns. c. Concatenated maximum likelihood phylogenetic tree of contigs encoding ribosomal proteins $(\geq 5)$ from across sponge metagenomic assemblies in the context of bacterial reference taxa. The tree is rooted by an archaeal outgroup. The metagenomic origin of each sequence is indicated by the dot plot, with lines corresponding to samples in the following order: P_S1, P_S2, P_S3, O_S4.1, O_S4.2, O_S5, and X_S6. Bars indicate the relative coverage of each ribosomal protein-encoding contig in each metagenome. Phylum affiliation is indicated for each clade according to the colour legend in panel a. In addition, classes are indicated for Proteobacteria: Alphaproteobacteria $(\alpha)$, Betaproteobacteria $(\beta)$, Deltaproteobacteria $(\delta)$, Epsilonbacteria $(\varepsilon)$, and Gammaproteobacteria $(\gamma)$. See legend in panel a for phylum colour assignment and patterns corresponding to each sponge species. See Data S1 for sample information, Data S2 for amplicon OTUs, Data S3 for relative abundances and read counts, Data S4 for metagenomic ribosomal protein contigs and their corresponding coverage, and Data S5 for the uncollapsed ribosomal protein phylogenetic tree. 
microbial community composition seen in the SSU rRNA gene amplicon results. However, several phyla had lower (i.e., Bacteroides, Cyanobacteria, and Proteobacteria) and higher (i.e., Actinobacteria and Chlamydiae) relative abundances in metagenomes than amplicons, perhaps due to differences in SSU rRNA gene copy number (30). Overall, the metagenomes confirmed the chlamydial patterns seen in amplicon data, with three distinct chlamydiae lineages found in high relative abundance when Chlamydiae were present (P_S1, O_S4.1, and O_S4.2) (Figure 1c and Data S4-S5). This provides further support for transient associations of diverse chlamydiae with several sponge species.

\section{Genome-resolved metagenomics of sponges expands sequenced chlamydial diversity}

Metagenome-assembled genomes (MAGs) were obtained from each sponge metagenome assembly using differential coverage profiles and consensus results from several binning tools (Figure S1). This resulted in 106 medium to high quality MAGs (median 89\% completeness and 1.4\% redundancy) (Data S4). Chlamydiae MAGs were further collected, manually curated, and reassembled (from P_S1, O_S4.1, and O_S4.2; Figure S1). This resulted in 18 high-quality draft chlamydiae genomes with high contiguity (median 35 contigs), high completeness (median 98.7\%), and low redundancy (median 1.01\%) (Data S6). Exceptionally, Chlamydiae bacterium O_S4.1_1 and O_S1_54 were 99\% complete and retrieved on only three contigs each. Phylogenomic trees were then inferred to determine the placement of sponge-associated chlamydiae MAGs within the Chlamydiae phylum, using four subsets of concatenated marker proteins with chlamydial and outgroup species representatives (Figures S2-S3, and Data S6-S7). The obtained species tree topology was consistent across ML reconstructions and the placement of sponge-associated chlamydiae MAGs was highly supported (Figures 2a and S3). A Bayesian phylogenetic tree was also inferred using the smallest subset of marker proteins $(n=15)$, which included those that best resolved the phyla present in the dataset (Figure S2 and Data S7). Here, species topology was overall consistent with the ML trees, with a few exceptions for longbranching taxa.

Sponge-associated chlamydiae MAGs were placed in four distinct chlamydial families (Figures $2 \mathrm{a}$ and S3). Interestingly, MAGs with a high level of relatedness were obtained from P_S1, O_S4.1, and O_S4.2, corresponding to the three highly abundant chlamydial lineages identified in amplicon and metagenomic analyses (Figures 1 and 2a). Two MAGs affiliated with the recently described 
117 Metagenomic Chlamydial Family D (MCF-D) (26), which includes a MAG from the glass sponge

118 Vazella pourtalesii (31), and indicates more widespread association of this family with sponges

119 (Figure 2a). Three additional MAGs and Chlamydiae bacterium 1063924-70 (26) formed a well-

120 supported clade with Simkaniaceae from marine, coastal, and host-associated environments

121 (Figures 2a and S3, and Data S6) (26, 32, 33). However, five other MAGs consistently form a

122 group sister to Simkaniaceae with several uncharacterized freshwater chlamydiae (Figures 2a and

123 S3, and Data S6) $(26,34)$. Based on its well-supported phylogenetic position and conserved gene

124 content, we propose this Simkaniaceae-like sister clade as a new chlamydial family Candidatus

125 Parasimkaniaceae (Figures 2 and S4-S5). In ML phylogenies a few long-branching taxa, including

126 one sponge-associated MAG (Chlamydiae bacterium O_S4.2_37), clustered together with

127 Simkaniaceae, yet were part of the Parasimkaniaceae with high support in the Bayesian tree

128 (Figures 2a and S3).

129 The remaining seven sponge-associated chlamydiae MAGs formed a clade with chlamydiae

130 from marine sediments (22) and fungal mycelium (26) that shares a common ancestor with

131 Chlamydiaceae and was previously referred to as Chlamydiae Clade IV (Figures 2a and S3) (22).

132 To reflect its consistent phylogenetic position, we propose to name this family Candidatus

133 Sororchlamydiaceae on the basis of improved taxon sampling here, distinct genomic characteristics

134 such as genome size, and conserved gene content (Figures 2b and S4-S5). Identifying sponge-

135 associated Sororchlamydiaceae is also interesting from an evolutionary perspective.

136 Sororchlamydiaceae shares a common ancestor with exclusively animal-associated chlamydiae

137 including Clavichlamydia salmonicola, a fish pathogen (35), and the Chlamydiaceae family, that

138 have thus far only been obtained from tetrapods (e.g., mammals, birds, and reptiles) (36). If many

139 Sororchlamydiaceae are indeed sponge symbionts, this could indicate an ancestral association of

140 these chlamydiae from the Chlamydiales order with metazoa, and subsequent long-term evolution

141 with animal hosts.

142 The observed differences in the relative abundance of Chlamydiae across the sponge species

143 was unexpected, given that healthy sponges typically have stable microbiomes (4). It was also

144 surprising that three phylogenetically distinct chlamydial lineages were present in these cases.

145 These chlamydiae could be actively acquired from the surrounding water by the filtering action of

146 the sponge. Conversely, in contrast to most other chlamydiae $(22,27)$, Sororchlamydiaceae

147 genomes also encode flagellar genes (Data S8), which could be used to encounter new hosts. 
a

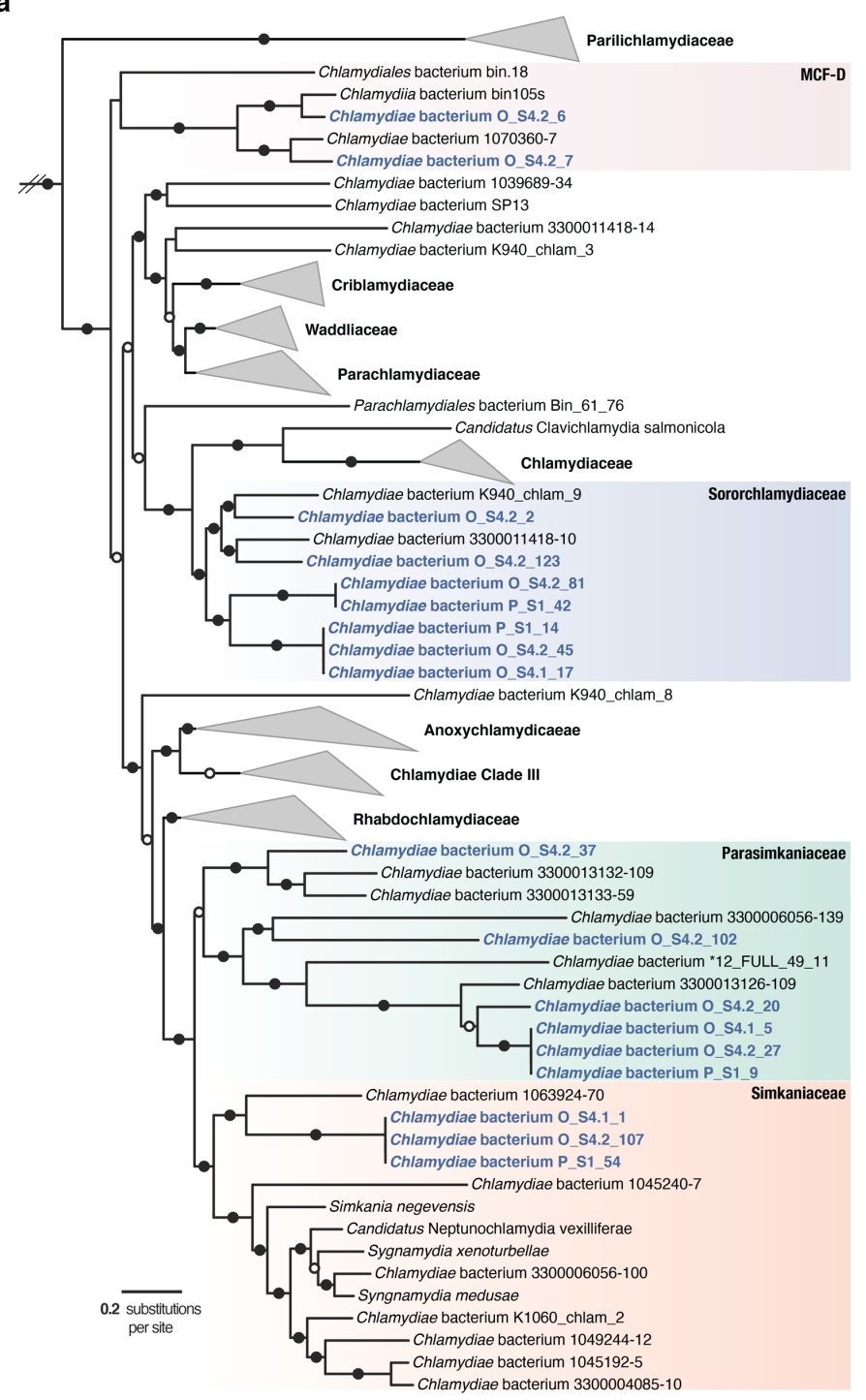

b

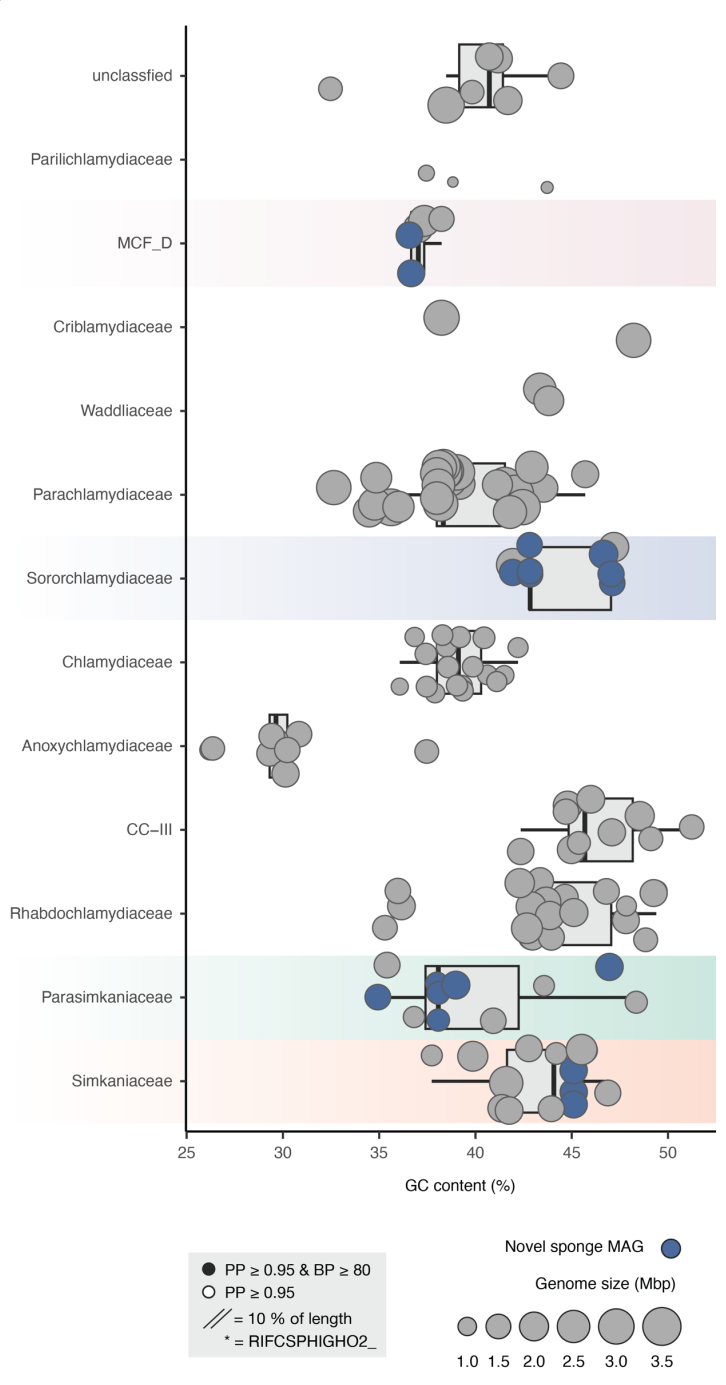

Figure 2. Chlamydiae metagenome-assembled genomes (MAGs) retrieved from sponges are phylogenetically diverse. a. Concatenated Bayesian phylogeny of Chlamydiae species relationships inferred using 15 marker gene NOGs (37) under the $\mathrm{CAT}+\mathrm{GTR}+\Gamma 4$ model of evolution. Branch support is indicated by coloured circles and includes posterior probability (PP) from the Bayesian inference and non-parametric bootstrap support (BP) from a maximum-likelihood reconstruction of the same dataset inferred with the PMSF approximation (38) of the $\mathrm{LG}+\mathrm{C} 60+\mathrm{F}+\mathrm{R} 4$ model of evolution (Data S5). Chlamydiae family names are indicated and those not including sponge-associated chlamydiae MAGs collapsed. Sponge-associated chlamydiae MAGs retrieved in this study are highlighted in blue. The tree is rooted by a PVC outgroup (not shown). See Figure S3 and Data S5 for additional species trees and uncollapsed phylogenies. b. Genome characteristics of sponge MAGs (blue) in the context of chlamydiae species representatives (grey). Boxplots indicate the distribution of GC content across different chlamydial families, with the area of circles indicating genome size. See Data S6 for genome characteristics. 
148 As part of their conserved lifestyles, chlamydiae have both an intracellular dividing phase, and an

149 extracellular non-dividing phase as elementary bodies (19). Chlamydiae can also enter states of

150 persistence inside their host cell when under environmental stress, where they then refrain from

151 dividing (19). As sponge-associated chlamydiae encode genes for the typical chlamydial lifecycle

152 (Data S8; see below), it is possible that they only increase in abundance under specific

153 environmental conditions, and otherwise remain as elementary bodies or in persistence states.

\section{Similarity in gene content of sponge-associated and environmental chlamydiae}

154 We compared the presence of central metabolic pathways across Chlamydiae and sponge155 associated MAGs to see if they resembled previously sequenced groups in terms of core 156 metabolism. Metabolic profiles of sponge-associated chlamydiae did not resemble known animal 157 pathogens (i.e., Chlamydiaceae and Parilichlamydiaceae), and instead their gene content was 158 similar compared to other members from the same chlamydial family (Figures S4-S5). Generally, 159 patterns also were similar across these chlamydial families, although Sororchlamydiaceae and 160 MCF-D genomes encode more genes for de novo biosynthesis of coenzymes and the amino acid 161 asparginine, and most Sororchlamydiaceae for pyrimidines (Figure S5 and Data S9). Overall, our 162 results mirrored prior findings (16, 24), with environmental chlamydiae (i.e., 163 Amoebachlamydiales) genomes encoding greater metabolic potential, and genomes from the 164 Chlamydiaceae and Parilichlamydiaceae encoding less, consistent with their highly specialized 165 lifestyles as animal pathogens (Figure S5 and Data S9).

166 Despite generally conserved core metabolism, some pathways are more common in spongeassociated genomes, such as de novo biosynthesis of aromatic amino acids (e.g., tryptophan) in 168 sponge-associated Simkaniaceae (Figure S5 and Data S9). It has been suggested that members of 169 the sponge microbiome exchange aromatic amino acids (39) and sponge-associated Simkaniaceae 170 may likewise do so or provide them to the sponge host. Similarly, most sponge-associated 171 Parasimkaniaceae encode a sodium-transporting NADH dehydrogenase, which is absent in all 172 other members of the family (Figure S5 and Data S9). Conversely, the first three genes of the TCA 173 cycle (i.e., citrate synthase, aconitase, and isocitrate dehydrogenase) are absent in most sponge174 associated Parasimkaniaceae genomes and present in other family members (Data S9). These genes 175 are also absent in Chlamydiaceae, which depend on the uptake of host-derived TCA cycle 176 intermediates (16) and this could likewise be the case for sponge-associated Parasimkaniaceae. 
177 Chlamydial families share a large proportion of gene content, with few genes unique to sponge178 associated lineages and more genes shared between closely related lineages (Figure S4). Thus, the 179 sponge-associated chlamydiae accessory genome corresponds more to phylogenetic affiliation than 180 to an ecological association with sponges. This could likewise indicate that sponge-associated 181 chlamydiae have similar lifestyles to close environmental relatives. Supporting this, sponge182 associated chlamydiae encode hallmark genes associated with endosymbiosis and the typical 183 chlamydial lifecycle that have been found conserved across Chlamydiae $(22,26)$. These include 184 nucleotide transporters (NTTs) that can be used for energy parasitism of ATP, the UhpC transporter 185 that can be used to uptake host-derived glucose-6-phosphate, the transcription factor EUO that acts 186 as the master regulator of the chlamydial biphasic lifecycle, and a type III secretion system that can 187 be used to mediate host interactions through the secretion of effectors $(16,18)($ Data S9). The 188 presence of these genes strongly suggests that sponge-associated chlamydiae are likewise 189 symbionts with the potential for an endosymbiotic lifestyle within the sponge host. Furthermore, 190 the metabolic profiles of sponge-associated chlamydiae are inconsistent with known chlamydial 191 pathogens of animals, suggesting the potential for host-beneficial interactions.

\section{degradative capacity indicate sponge-associated chlamydiae engage in sponge interactions}

192 Genes more commonly found in sponge-associated chlamydiae genomes were further investigated 193 and revealed degradative capacities consistent with a sponge-symbiont lifestyle and the use of host194 derived compounds (Figures S4-S6, and Data S8-S9). For example, all Sororchlamydiaceae 195 genomes encode taurine dioxygenase (TauD) (Figures S6 and S8). This enzyme degrades taurine 196 to sulfite and is widespread among members of the sponge microbiome who can use it to degrade 197 host-derived taurine (40). Taurine is present in, and released from, nearly all marine metazoans

198 (41). The MAGs Chlamydiae bacterium S1_14, S4.1_17, and S4.2_45, which correspond to the 199 abundant Sororchlamydiaceae lineages (Figures 1c and 2a), also encode a putative scyllo-inositol 200 2-dehydrogenase (IolW), an enzyme that can degrade scyllo-inositols (Figure S6 and Data S8). 201 Similarly, the abundant Simkaniaceae sponge MAGs, Chlamydiae bacterium S1_54, S4.1_1, and 202 S4.2_107 (Figures 1c and 2a), have the genetic potential to degrade xylose using the concerted 203 action of xylose isomerase (XylA) and xylulokinase (XylB) (Figure S6 and Data S8). Genes for 204 degrading scyllo-inositol and xylose are rare or absent in other chlamydiae genomes (Figure S6 205 and Data S8). However, known sponge symbionts do encode these genes as well, such as 
206 Poribacteria, a candidate phylum so far only found to reside in the sponge extracellular matrix (42-

207 44). Inositols are common in eukaryotes and rare in bacteria, and the sponge host has been 208 suggested as the source of inositols for inositol-degrading sponge-associated bacteria (43-45).

209 Several typically-eukaryotic genes were also identified in some sponge-associated chlamydiae 210 genomes and further suggest host-interactions (Figure S6 and Data S8). These genes include sterol 211 reductases identified in Sororchlamydiaceae and sponge-associated Simkaniaceae, and carnitine 212 O-acetyltransferase in sponge-associated Simkaniaceae (Figure S6 and Data S8). Sterol reductases

213 perform the final step in ergosterol or cholesterol biosynthesis and have previously been found in 214 intracellular bacteria, including several chlamydiae (46). In phylogenetic trees of these sterol 215 reductases (K00223/K00213 and K09828), chlamydial sequences branch together with both 216 bacterial and eukaryotic homologs, and could have been acquired by horizontal gene transfer 217 (HGT) from either (Data S5). Carnitine O-acetyltransferase is used in eukaryotes to transport 218 carnitine across the mitochondrial matrix (47), and sponge-associated chlamydiae may use it to 219 obtain carnitine from a sponge host. Carnitine is abundant in animal tissues, and in bacteria it is 220 used as an osmoprotectant or metabolized (47). Genes related to carnitine degradation have been 221 found in members of the sponge microbiome, and carnitine is also abundant in the sponge 222 extracellular matrix (48). In a phylogenetic tree of carnitine O-acetyltransferase (K00624) 223 chlamydial sequences branch within eukaryotic homologs, suggesting that it was obtained by HGT 224 from a eukaryotic host (Data S5).

225 We also identified genes indicating that several sponge-associated chlamydiae can degrade 226 acetoin, possibly derived from other members of the sponge microbiome, as a carbon and energy 227 source (Figure S6 and Data S8). Acetoin is a volatile organic compound that some bacteria can use 228 as an energy and carbon storage compound, and which can act as a sole carbon source under 229 glucose-limitation (49, 50). Most Sororchlamydiaceae, MCF-D, and sponge-associated 230 Simkaniaceae appear to be acetoin-degrading bacteria as they encode an acetoin dehydrogenase 231 complex (AcoA-B) (Figure S6). These genes are rare in other chlamydiae and largely absent in 232 Parasimkaniaceae (Figure S6). The AcoA-B complex is used to degrade acetoin to acetaldehyde 233 and acetyl-CoA, with the concerted reduction of $\mathrm{NAD}^{+}$to $\mathrm{NADH}$ (49-51). Acetaldehyde can then 234 undergo further fermentation to ethanol by alcohol dehydrogenases, such as those found in 235 Sororchlamydiaceae and MCF-D, acetyl-CoA can enter the TCA cycle, and NADH can be shuttled 236 into the electron transport chain or used as a reducing agent in other reactions (Figure S6). Some 
237 sponge-associated Simkaniaceae and Sororchlamydiaceae may also be able to produce acetoin 238 through the fermentation of pyruvate to acetolactate using Acetolactate synthase (Figure S6). 239 Acetolactate is then converted to acetoin spontaneously under aerobic conditions or through the 240 action of acetolactate decarboxylase (49), which is encoded in some sponge-associated 241 Simkaniaceae genomes (Figure S6).

242 In addition, we identified genes suggesting that some sponge-associated chlamydiae are 243 involved in degrading both organic pollutants and toxic compounds produced by members of the

244 sponge microbiome. A putative S-(hydroxymethyl)glutathione dehydrogenase gene was found in 245 several sponge-associated Sororchlamydiaceae and MCF-D MAGs, and in a few other chlamydial 246 groups (Figure S6 and Data S8). This enzyme is involved in oxidizing formaldehyde, a toxic 247 compound that can, for example, be produced as an intermediate during methylotrophy (52), for 248 which genes have been found expressed by sponge symbionts (53). We also identified several 249 genes involved in chlorocatechol and catechol degradation (i.e., dienelactone hydrolase, 3250 oxoadipate enol-lactonase, and catechol 2,3-dioxygenase) (Figure S6 and Data S8). Anthropogenic 251 contamination is a growing concern in marine ecosystems, and microbially-mediated degradation 252 of such organic pollutants and other aromatics reach a branching point at chlorocatechol and 253 catechol (54). Genes involved in these degradative pathways have been found in members of the 254 sponge microbiome, and may also be used to detoxify compounds produced by other microbial 255 members (55). Dienelactone hydrolases perform a key step in the modified ortho-cleavage pathway 256 by degrading dienelactones to maleylacetate, which can then be further catabolised before entering 257 the TCA cycle $(56,57)$. Sponge-associated Simkaniaceae encode dienelactone hydrolase (Figure 258 S6), and genes with the dienelactone hydrolase family protein domain (PF01738) are found across 259 sponge-associated Sororchlamydiaceae genomes (Data S8). These sponge-associated chlamydiae 260 may use these genes to protect themselves, or the sponge host, from these toxic compounds, 261 whether they originate from external sources or other microbial community members.

\section{Widespread potential for secondary metabolite biosynthesis across the phylum Chlamydiae}

262 Sponges are well-known as reservoirs for the discovery of natural products with medical and 263 industrial importance, many of which are secondary metabolites produced by microbiome 264 members (6-8). Typically, genes for producing secondary metabolites are organized in biosynthetic 265 gene clusters (BGCs) (7). Recently, metagenomic analyses have revealed that BGCs are found 
a

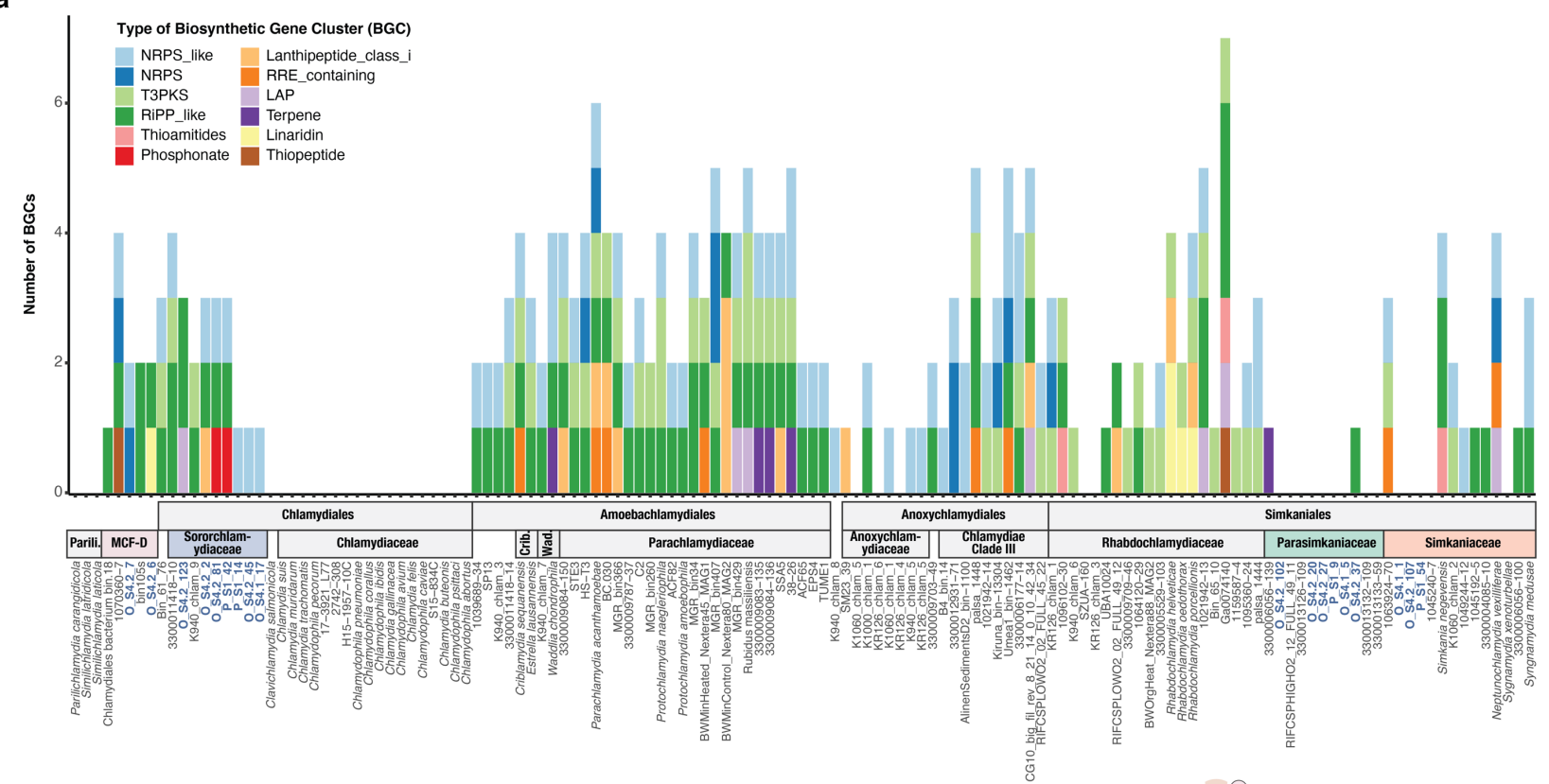

C

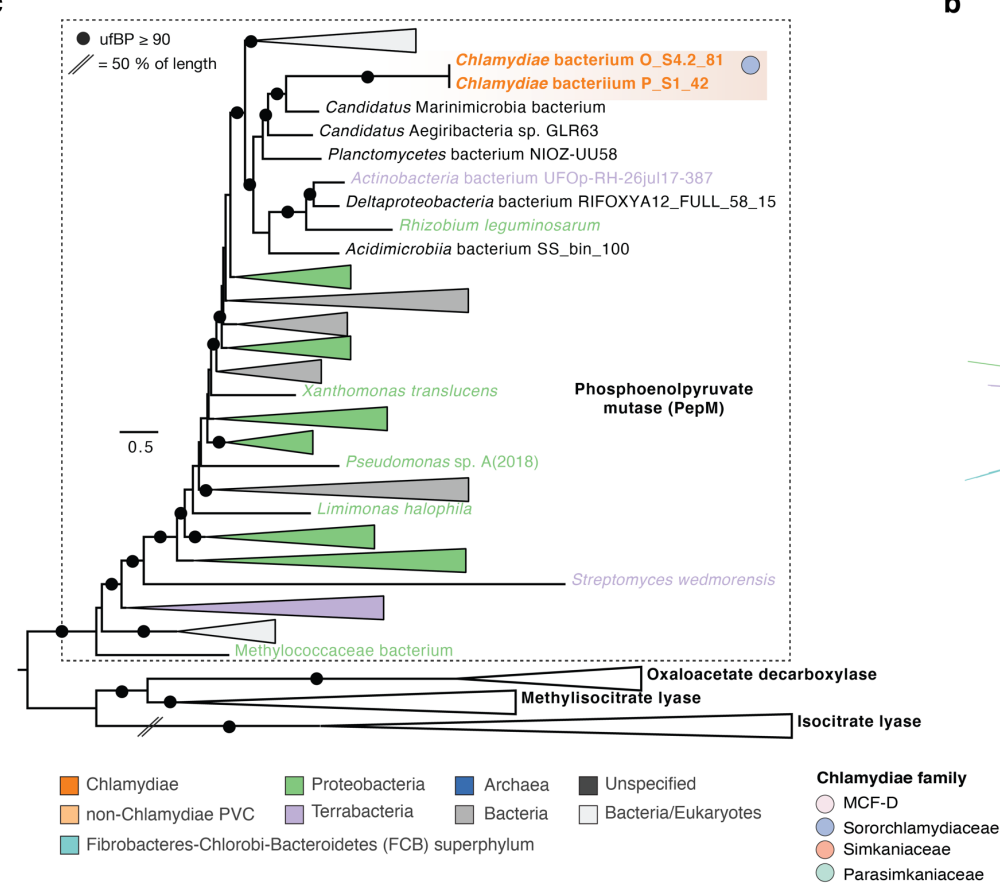

b

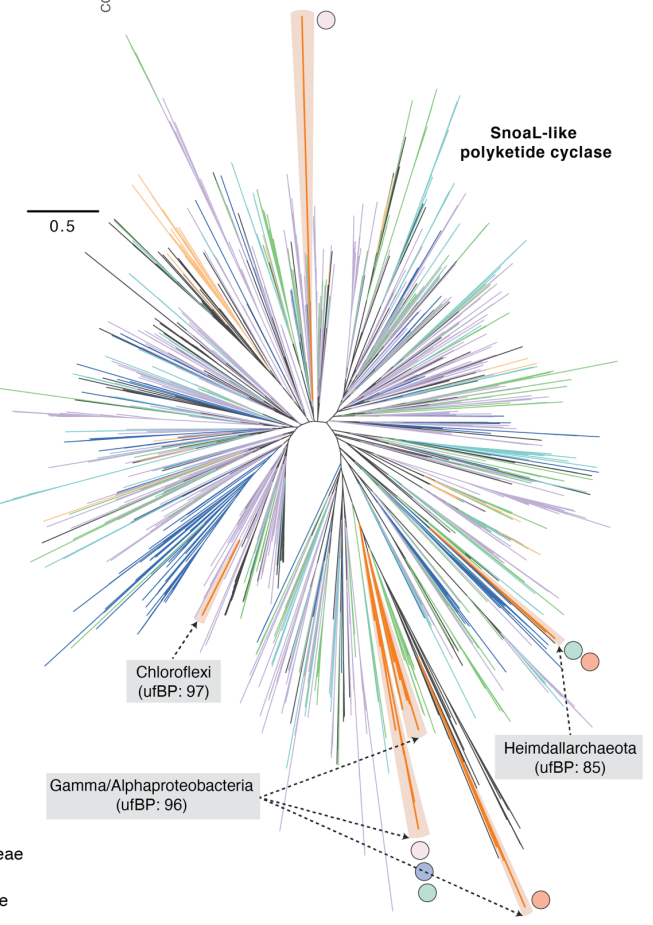

Figure 3. Biosynthetic gene clusters (BGCs) are found across Chlamydiae and sponge-associated chlamydiae genomes. a. The number and type of BGCs (in $>1$ genome) identified across Chlamydiae using antiSMASH. Order and family classification is indicated alongside shortened species names. See Data S10 for a full overview of BGCs. Maximum-likelihood phylogenies of a group of polyketide cyclases (SnoaL-like; PF07366) (b), which are involved in secondary metabolite biosynthesis, and of Phosphophenolpyruvate mutase (PepM; PF13714) (c), which performs the first step in phosphonate biosynthesis. Ultrafast bootstrap support values are indicated by a black circle (c). Branch and clade colours indicate taxonomy according to the legend $(\mathbf{b}, \mathbf{c})$. The presence of chlamydiae families including sponge-associated chlamydiae genomes is indicated by the coloured circles, with the taxonomy of the sister clade indicated where supported (c). See Data S5 for uncollapsed phylogenies and sequence accessions. 
across a broad phylogenetic range of bacteria in sponge microbiomes (58). To gain further

perspective on the genetic capacity for secondary metabolite biosynthesis across Chlamydiae, we used antiSMASH (59) to investigate BGCs in representative genomes and our sponge-associated chlamydiae MAGs (Figure 3 and Data S10). Unexpectedly, our analysis revealed that BGCs are widespread across the phylum Chlamydiae, with many chlamydial genomes encoding multiple and different types of BGCs, most with unknown functions (Figure 3a and Data S10).

272 Genomes of Sororchlamydiaceae and Amoebachlamydiales encode the most conserved set of 273 BGCs, with many encoding NRPS-like (non-ribosomal peptide synthetase), RiPP-like 274 (Ribosomally-synthesized and post-translationally modified peptides), and T3PKS (type III 275 polyketide synthase) BGCs (Figure 3a). However, fewer BGCs were identified in 276 Anoxychlamydiaceae, were largely absent in Parasimkaniaceae and sponge-associated 277 Simkaniaceae, and completely absent in all Chlamydiaceae and Parilichlamydiaceae genomes 278 (Figure 3a). We found additional PKS and NRPS genes, which are central in the biosynthesis of 279 various secondary metabolites, in sponge-associated chlamydiae MAGs. MCF-D member 280 Chlamydiae bacterium S4.2_7 encodes NRPS gene homologs related to those found in Simkania negevensis (Data S8). One group of PKS genes, SnoaL-like polyketide cyclases (PF07366), were found across many sponge-associated chlamydiae MAGs (Data S8). Phylogenetic analysis of this protein family showed that it has been gained multiple times by different chlamydial groups and from diverse potential HGT partners (Figure 3b). Despite BGCs not being widely identified in Parasimkaniaceae, some genomes do encode this PKS gene.

Two Sororchlamydiaceae sponge MAGs (Chlamydiae bacterium S1_42 and S4.2_81) encode a phosphonate BGC (Figure 3a), with closest homology to BGCs used to produce the antibiotic fosfomycin (Data S10). Fosfomycin inhibits bacterial cell wall biosynthesis by binding to the active site of MurA, which performs the initial step in peptidoglycan biosynthesis (60). Phosphoenolpyruvate mutase (PepM) performs the first committed step for synthesizing fosfomycin and other phosphonates (61). In a phylogenetic tree, chlamydial PepM homologs

292 formed a well-supported clade together with known PepM sequences, indicated that they likely 293 have the same function (Figure 3c). However, we were unable to determine the HGT donor, since 294 chlamydial homologs formed a clade with diverse bacteria, primarily represented by MAGs (Figure 295 3c). Interestingly, Chlamydia spp. are resistant to very large quantities of fosfomycin due to 296 conserved changes in the MurA active site (62). Further investigation into MurA sequence 
297 evolution and the potential for fosfomycin production across chlamydiae could elucidate whether

298 Chlamydiaceae MurA resistance may be connected to an ancestral capacity for fosfomycin 299 production.

300 Overall, our results show that many chlamydiae have the potential to produce secondary metabolites, which may play a previously unrecognized role in their endosymbiotic lifestyles. As

302 far as we are aware, secondary metabolite biosynthesis had not been previously noted or 303 investigated in chlamydiae. This could be explained by the absence of these gene clusters in the 304 most-studied chlamydial animal pathogens (i.e., Chlamydiaceae and Parilichlamydiaceae).

305 Chlamydiae is part of the PVC superphylum and other PVC members are also associated with 306 sponges $(13,29)$. In particular, Planctomycetes have been identified as a potential reservoir of 307 novel secondary metabolites $(63,64)$. Despite Planctomycetes having substantially larger genomes 308 (64), comparable numbers of BGCs were identified in Chlamydiae genomes (Figure 3a). The 309 Chlamydiae phylum may thus likewise represent a reservoir for the discovery of secondary 310 metabolites. Based on their endosymbiotic lifestyles and smaller genome sizes, it was surprising to 311 identify BGCs as common in many chlamydiae genomes. These chlamydial BGCs could function 312 in inter-microbial warfare, in communication, or in mediating host-interactions (65).

313 In addition, chlamydial BGCs could function in providing chemical defence to the host. 314 Members of the sponge microbiome have been suggested to provide chemical defence to the 315 sponge host (5). Recently, an endosymbiosis was identified between a Haliclona sponge species 316 and a gammaproteobacteria mediated by chemical defence through antibiotic production (66). 317 Sponge-associated chlamydiae that encode BGCs (i.e., Sororchlamydiaceae and MCF-D) may 318 likewise participate in host-beneficial defensive endosymbioses. MAGs for other typically 319 endosymbiontic bacteria, including Legionellales and Rickettsiales, were also obtained from the 320 metagenomes (Data S4). This suggests the potential for antagonistic intracellular interactions 321 between co-infecting endosymbionts, perhaps mediated by secondary metabolites. Some protist322 infecting Parachlamydiaceae have previously been shown to be mutualists that protect their host 323 amoeba against Legionella infection through an unknown mechanism $(67,68)$. This mechanism 324 may have a basis in the production of secondary metabolites as many BGCs were identified in 325 Parachlamydiaceae. Some sponge-associated chlamydiae could offer similar defensive benefits 326 again co-infection to their host. Interestingly, novel antimicrobial compounds have also been 
327 isolated from sponges that are active against chlamydial species (69), and could indicate

328 antagonistic interactions with the sponge host or members of the sponge microbiome.

\section{Chlamydiae are associated with other sponge species and marine invertebrates}

To determine whether sponge-associated chlamydiae are associated with other hosts or 330 environments we screened publicly available SSU rRNA gene amplicon datasets for close relatives

331 ( $\geq 95 \%$ identity) (Figure $4 \mathrm{a}$ and Data S11). Relatives of sponge-associated chlamydiae were found 332 almost exclusively in marine environments, with higher prevalence in marine invertebrates such as 333 sponges, corals, sea squirts, and molluscs (Figure 4a). MCF-D was present in a higher proportion

334 and wider range of environments, yet still primarily of marine origin. Unfortunately, no SSU rRNA

335 gene was obtained for any of the sponge-associated Parasimkaniaceae MAGs, and their environmental distribution could not be assessed. Despite the clear association with marine environments here, other chlamydiae have also been found associated with sponges from

338 freshwater lakes (70). Relatively few environments were identified with higher abundances of 339 sponge-associated chlamydiae relatives (Data S11), possibly due to chlamydiae being undetected

340 and underestimated by many common primers for surveying microbial diversity (20-22). Those 341 identified included incubations of Great Barrier Reef lagoon water (71), and humic acid amended 342 aquaculture systems (Data S11). In addition to our study, and the previous study of these sponge 343 species (15), Chlamydiae have also previously been found in high relative abundance in association 344 with sponges. This includes Suberites zeteki, a marine sponge invasive in Hawaii (14), and $H$. 345 panicea, but only after ex situ cultivation (72).

346 Although chlamydiae have been found across different sponges and relatives of sponge347 associated chlamydiae were detected in various marine invertebrates, it is possible that they instead 348 have another sponge-associated eukaryotic host. To help elucidate this, eukaryotic SSU rRNA 349 genes across the metagenomes were collected and classified (Figure 4b and Data S4). Some of the 350 identified eukaryotes are present in multiple samples. However, importantly, no other eukaryotes 351 apart from the sponge were found across the samples with high chlamydiae relative abundances 352 (Figure 1 and $4 \mathrm{~b}$ ). Still, additional eukaryotes may have been missed in the metagenomes, for 353 example due to DNA extraction bias. We did identify a mitochondrial cytochrome c oxidase 354 subunit 1 (CO1) gene most closely related to the green algae Picochlorum in samples with high 355 chlamydiae relative abundances, and this could represent an alternative host (Data S4). 
a

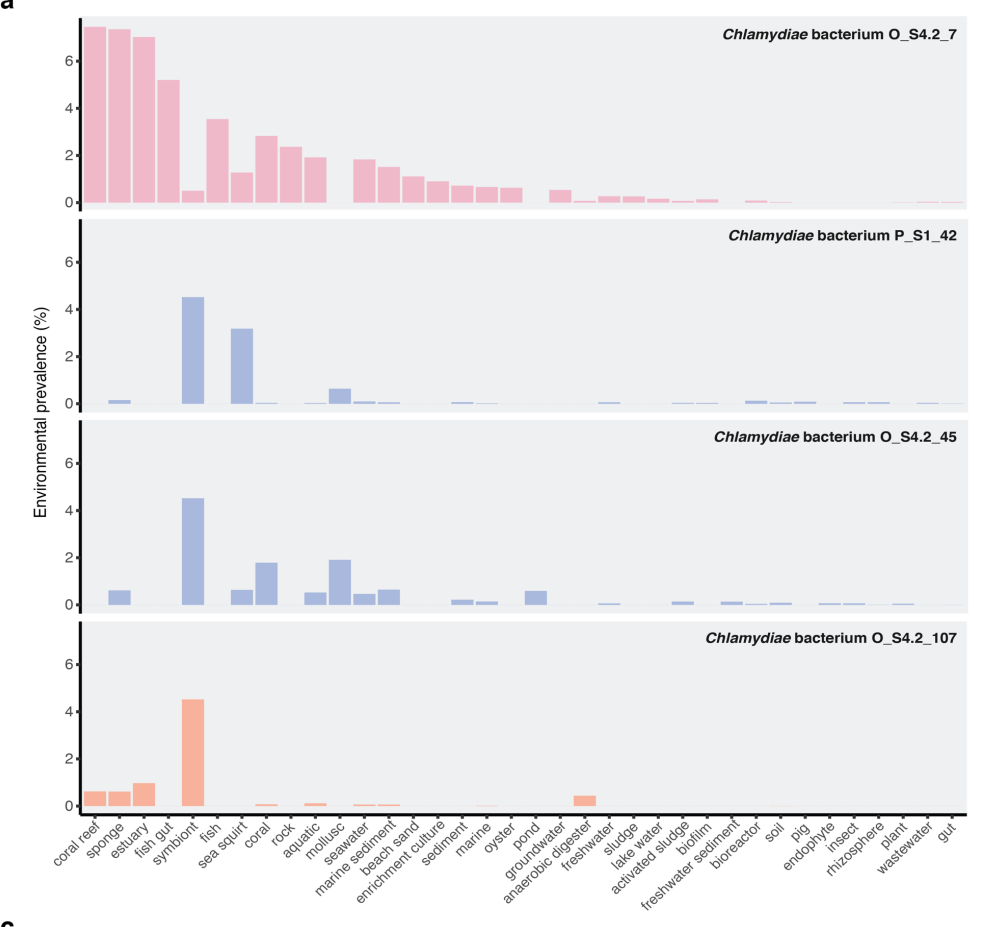

b

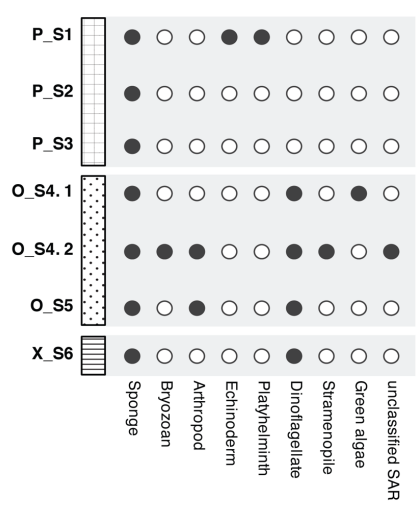

Sponge species

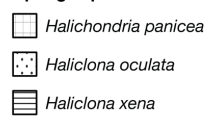

Chlamydiae family

MCF-D

Sororchlamydiaceae

Simkaniaceae

Parasimkaniaceae

c

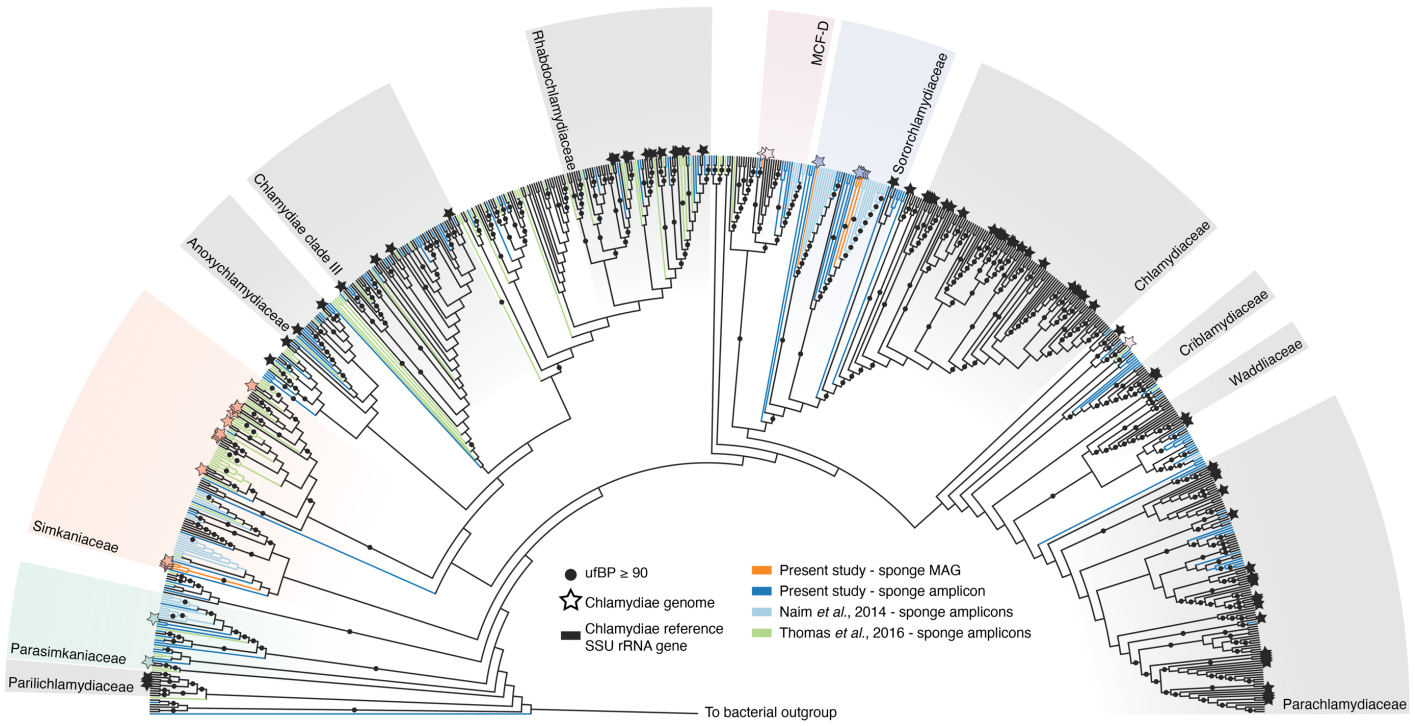

Figure 4. Relatives of sponge-associated chlamydiae MAGs are primarily found in marine habitats, and no other putative eukaryotic hosts were identified in the sponge metagenomes. a. Percentage of amplicon samples from various environments with SSU rRNA genes $\geq 95 \%$ identity to the indicated sponge MAG. A representative from each phylogroup with MAGs containing SSU rRNA genes is shown. Only environments with $\geq 100$ samples, and clear labels are shown. See Data S11 for full IMNGS (73) results of SSU rRNA gene searches against SRA amplicon datasets. b. Presence of eukaryotic SSU rRNA genes, and their corresponding taxonomy, across sponge sample metagenome assemblies. See Data S4 for full taxonomy, contig IDs, and contig coverage. c. Sponge-associated chlamydiae are restricted to specific chlamydial groups. Maximum-likelihood phylogeny of small subunit rRNA genes from Chlamydiae (and outgroup sequences) inferred using the GTR+F+R10 model and shown as a cladogram for clarity (See Data S5 for sequence accessions and branch lengths.). Included are sequences from reference chlamydiae (black), sponge-associated chlamydiae genomes and amplicons from the present study (orange and blue), chlamydiae amplicons previously obtained from these sponge species (light blue; Naim et al., 2014), and chlamydial amplicons obtained from a prior study of sponge microbial diversity (green; Thomas et al., 2016). Sequences corresponding to chlamydiae genomes, and sequenced diversity, are indicated with stars. Chlamydial families are coloured and labelled. 
Chlamydiae were found across a wide range of sponge microbiomes in a recent amplicon survey (13). This prompted us to examine whether specific chlamydial groups are associated with sponges.

358 To answer this question we inferred a SSU rRNA gene phylogeny using chlamydial sequences 359 obtained from this wide survey (13), from our study, and from the prior study of these sponge

360 species (15), in the context of a representative dataset of bacterial and chlamydial SSU rRNA gene 361 sequences $(20,21)$, and sequenced chlamydiae species representatives (Data S6). In the resulting 362 phylogenetic tree, a striking separation became apparent, with the vast majority of sponge363 associated sequences affiliating with less studied chlamydial families (Figure 4c). No sponge 364 sequences grouped together with the well-studied Chlamydiaceae animal pathogens, and few 365 sequences affiliated with the protist-infecting Amoebachlamydiales families (i.e., 366 Criblamydiaceae, Waddliaceae, and Parachlamydiaceae) (Figure 4c). As expected, sequences from 367 the present study and previous study of these sponge species (15) clustered together in 368 Sororchlamydiaceae, MCF-D, Simkaniaceae, and Parasimkaniaceae (Figure 4c). Chlamydial 369 sequences from the wide survey of sponge microbiomes (13) primarily grouped together with 370 Parasimkaniaceae, Simkaniaceae, Rhabdochlamydiaceae, and in unclassified groups (Figure 4c). 371 Altogether, these observations provide evidence for widespread associations between less-studied 372 chlamydial groups and sponges.

\section{Conclusions and future perspectives}

373 Using genome-resolved metagenomics we have expanded sequenced chlamydiae with 18 high374 quality genomes that provide insight into chlamydial associations with animal hosts. All cultivated 375 chlamydiae are obligately intracellular (18) and based on their gene content sponge-associated 376 chlamydiae are likely endosymbionts. However, direct confirmation is needed. Given that sponge377 associated chlamydiae are capable of acquiring carbon and energy directly from eukaryotic hosts 378 (e.g., through the action of NTTs and UhpC etc.), it is surprising that they have the capacity to 379 degrade a wide range of sponge-derived compounds and compounds present in the larger microbial 380 community. The presence of genes for degrading toxins and pollutants, and BGCs in some sponge381 associated chlamydiae could suggest host-beneficial effects. More generally, our findings open the 382 door for further exploration of BGCs in Chlamydiae, and suggest larger roles for secondary 383 metabolites in endosymbiotic interactions. Relatives of sponge-associated chlamydiae are 384 prevalent in other sponges and marine invertebrates, where they have unknown effects. 
We found that sponge-associated chlamydiae vary in their relative abundance across specimens of the same sponge species. This points to important unanswered questions about the nature of sponge-chlamydiae associations. Foremost, it is currently unclear whether the presence of chlamydiae is beneficial or detrimental to the sponge, and if their presence potentially represents an indicator for environmental conditions and host health? Future studies are also needed to confirm whether chlamydiae associate directly with the sponge host as symbionts, or if their interaction is secondary through another eukaryotic host. Altogether, our work represents a first step in untangling the potentially wide impacts of chlamydiae on marine ecosystems.

\section{Description of Candidatus Sororchlamydiaceae fam. nov.}

393 (So.ror.chla.my.di.a.ce'ae. L. fem. n. soror sister; Chlamydiaceae taxonomic name of a bacterial 394 family; L. suff. -aceae ending to denote a family; Sororchlamydiaceae referring to the close relationship to the bacterial family Chlamydiaceae)

396 The family Candidatus Sororchlamydiaceae (formally Chlamydiae Clade IV) represents a distinct monophyletic lineage, sister to Chlamydiaceae, supported by concatenated marker protein

398 phylogenies in the present study (Figures 2a and S3) and in prior work (22, 26).

399 Sororchlamydiaceae share conserved gene content and metabolism, and have larger genome sizes 400 and greater metabolic potential than Chlamydiaceae members (Figures 2b and S4-S6) (22).

\section{Description of Candidatus Parasimkaniaceae fam. nov.}

401 (Par.a.sim.ka.ni. a.ce'ae. Gr . prep. para alike, alongside of; N.L. fem. n. Simkania taxonomic name 402 of a bacterial genus; L. suff. -aceae ending to denote a family; Parasimkaniaceae referring to the 403 close relationship to the bacterial family Simkaniaceae)

404 The family Candidatus Parasimkaniaceae represents a distinct monophyletic lineage supported by 405 concatenated marker protein phylogenies that is closely related to the Simkaniaceae family (Figures 406 2a and S3). Parasimkaniaceae share conserved gene content, and have on average smaller genome 407 sizes and reduced metabolic potential relative to Simkaniaceae members (Figures 2b and S4-S6). 


\section{MATERIALS AND METHODS}

\section{Sponge collection and DNA extraction}

409 Six sponge specimens from three sponge species (Halichondria panicea - P_S1, P_S2, and P_S3;

410 Haliclona oculata - O_S4 and O_S5; Haliclona xena - X_S6) were collected from the

411 Oosterchelde estuary in the Netherlands, washed in autoclaved seawater, and stored at $-80{ }^{\circ} \mathrm{C}$

412 (Figure S1 and Data S1). Several were sampled alongside those investigated in a previous study

413 (15). The sponge SSU rRNA gene from each metagenome was used to confirm sponge species 414 identification (Data S1).

415 For generating SSU rRNA gene amplicons, DNA was extracted from $0.2 \mathrm{~g}$ of each 416 specimen using the DNAeasy PowerLyser Powersoil Kit according to manufacturer's protocols, 417 with DNA elution in water and bead beating with the PowerLyzer 24 homogenizer at 4000 rpm for 41845 s. For O_S4 (O_S4.2), O_S5, and X_S5 this DNA was also used for metagenomic sequencing 419 (Figure S1). However, due to high levels of fragmentation an additional extraction optimized for 420 high-molecular-weight DNA was performed for metagenomic sequencing of P_S1, P_S2, P_S3 421 and O_S4 (O_S4.1) (Figure S1). Here, bead beating with the DNAeasy PowerLyser Powersoil Kit 422 was used as described above, but with the addition of $0.2 \mathrm{M}$ ethylenediamine tetraacetic acid 423 (EDTA) (1:1 ratio) prior to the lysis step to inhibit DNAses. After bead beating, 10\% cetyl 424 trimethylammonium bromide buffer (CTAB) (1:4 ratio), $0.5 \mathrm{M} \mathrm{NaCl}$ (1:2 ratio), 0.1 M EDTA (1:4 425 ratio), $10 \mu \mathrm{L} \beta$-Mercaptoethanol (100\%), and $5 \mu \mathrm{L}$ Proteinase K (600 mAU/mL) were added and 426 samples incubated overnight at $56{ }^{\circ} \mathrm{C}$. RNAse A was then added (final concentration of $0.3 \mathrm{ng} / \mu \mathrm{l}$ ) 427 and the sample incubated for $30 \mathrm{~min}$ at $37^{\circ} \mathrm{C}$. Two rounds of chloroform/Isoamylalcohol 24:1 (1:1 428 ratio) addition, incubation for $2 \mathrm{~min}$ at room temperature, centrifugation (10000 x g for $10 \mathrm{~min}$ ), 429 and transfer of the aqueous phase were then performed. DNA was precipitated using isopropanol 430 (6 hr) and pelleted with centrifugation for $15 \mathrm{~min}$ at $10000 \mathrm{x}$ g at $4{ }^{\circ} \mathrm{C}$, before being washed twice 431 with $80 \%$ ethanol and eluted in water.

\section{Generation of bacterial SSU rRNA gene amplicons}

433 A two-step PCR approach was used to obtain SSU rRNA gene fragments for amplicon sequencing 434 (Figure S1), using the bacterial-specific primers S-D-0564-a-S-15 (AYTGGGYDTAAAGNG) and 435 S-D-Bact-1061-a-A-17 (CRRCACGAGCTGACGAC) (74), that capture most chlamydial lineages 436 (22). HotStarTaq DNA Polymerase (QIAGEN) was used with the following reaction conditions: 437 initial heat activation at $95{ }^{\circ} \mathrm{C}(15 \mathrm{~min})$, followed by 28 cycles of denaturation at $94{ }^{\circ} \mathrm{C}(60 \mathrm{~s})$, a 
step-down to $70{ }^{\circ} \mathrm{C}(1 \mathrm{~s})$, a ramping rate of $0.4{ }^{\circ} \mathrm{C} / \mathrm{s}$ to $50{ }^{\circ} \mathrm{C}$ for annealing $(60 \mathrm{~s})$, and a ramping rate of $0.8{ }^{\circ} \mathrm{C} / \mathrm{s}$ to $72{ }^{\circ} \mathrm{C}$ for extension $(60 \mathrm{~s})$, with a final extension at $72{ }^{\circ} \mathrm{C}(10 \mathrm{~min})$. A second

440 PCR reaction was performed according to the manufacturers protocol to obtain sequence libraries

441 with adaptor sequences from the TruSeq DNA LT Sample Prep Kit (Illumina). PCR products were

442 purified using magnetic Agencourt AMPure XP beads (Beckman Coulter) and sequencing 443 performed on the Illumina MiSeq platform (2x300 bp).

444 Sequence reads were demultiplexing and quality control performed using cutadapt v. 1.10 (75)

445 to remove remaining adaptor and primer sequences, trim 3' read ends to a minimum Phred quality 446 score of 10, and remove reads shorter than $100 \mathrm{bp}$ in length. VSEARCH v. 1.11.1 (76) was then 447 used to merge forward and reverse reads (-fastq-minovlen 16), to de-replicate reads (448 derep_fulllength), and to obtain centroid OTU clusters at 97\% identity. Chimeric sequences were 449 detected and removed using UCHIME (77) with the SILVA123.1_SSUref_tax:99 database (78).

450 OTUs were taxonomically classified using the LCAClassifier from CREST-2.0.5 (79) (Data S2).

451 OTU relative abundance across samples are available in Data S3.

\section{Metagenome sequencing and assembly}

453 The Nextera DNA Library Prep Kit (Illumina) was used to prepare sequence libraries with $25 \mathrm{ng}$ 454 of input DNA, followed by sequencing with Illumina NovaSeq6000 System for P_S1, P_S2, P_S3 455 and O_S4 (O_S4.1), and with Ilumina HiSeq2500 System for O_S4 (O_S4.2), O_S5 and X_S6. 456 Quality control of resulting sequence reads was performed to remove adaptors and low-quality 457 sequences using Trimmomatic v. 0.35 (80) with the options: ILLUMINACLIP:TruSeq3458 PE.fa:2:30:10 LEADING:3 TRAILING:3 SLIDINGDOWN:4:15 MINLEN:50. Read quality was 459 assessed using FastQC v0.11.4 (81). Resulting paired sequence reads were then assembled using 460 MEGAHIT v3.13 (82) (--meta --only-assembler), and assembly statistics obtained with QUAST 461 v5.0.2 (83) (Data S1). Proteins sequences were predicted using prodigal v2.6.3 (84). Barrnap v. 0.9 462 (85) was used to identify metagenomic SSU rRNA genes, which were classified using the 463 LCAClassifier from CREST-3.1.0 (79) (Data S4).

\section{Binning of metagenome-assembled genomes}

465 Differential read coverage was obtained by mapping each set of sequence reads against each 466 assembled metagenome using Bowtie2 v2.2.6 (86). MAGs from each metagenome were obtained 467 using differential coverage binning with metaBAT 2.12.1 (87), CONCOCT v. 1.1.0 (88), and 468 MaxBin v. 2.2.7 (89) (Figure S1). The metaWRAP v. 1.2.4 "bin_refinement" module was then 
used to consolidate resulting bins into hybridized bin sets. The highest quality hybridized or original bin was selected with a cut-off of 70\% completeness and 10\% redundancy (90) (Data S4).

471 Several assemblies had smaller sizes (P1_S2 and P1_S3) and few MAGs above quality thresholds

472 were obtained for these (Data S1 and S4). Further manual refinement of chlamydiae MAGs was

473 performed using anvi'o v.6.2 (91, 92), followed by reassembly with the metaWRAP v. 1.2.4

474 "reassemble_bins" module (90), and additional manual curation (Figure S1).

\section{Genome characteristics and annotation}

476 Chlamydiae MAGs were annotated using Prokka v1.14.6 (93). In addition, protein-coding genes 477 were annotated with NCBI NR protein database (94) top blastp hits, and Pfam (95) and TIGRFAM

478 (96) domains identified by InterProScan 5.47-82.0 (97). Comparative genomic analyses were performed between chlamydiae MAGs obtained here and a set chlamydiae species representatives

480 with high-quality genomes. Genome characteristics and genome quality were determined with 481 MiComplete v. 1.1.1 (98) (Data S6) using a marker gene-set conserved in complete chlamydiae 482 genomes (22) (Data S6). Genes were also assigned to eggNOG 4.5 (37) NOGs at the universal483 level using eggNOG-mapper 1.0.3 (“-d NOG”) (99), and KEGG KOs (100) identified using 484 GhostKOALA (101) (chlamydiae representatives) and BlastKOALA (chlamydiae from the present 485 study). Identified COGs were also assigned to COG pathways (Data S8) (102). AntiSMASH 6 beta 486 (59) was used to identify BGCs, and top hits to MIBiG clusters (103) where found (Data S10).

\section{Ribosomal protein phylogeny of metagenomic contigs}

488 Microbial community composition was assessed by identifying metagenomic contigs encoding 489 ribosomal proteins (at least 5 of 15), typically found together in a conserved gene cluster (104), 490 using a previously described pipeline (105) (Data S4). Ribosomal proteins from each contig were 491 concatenated and a ML phylogeny inferred using RAxML 8.2 .4 (106) with the PROTCATLG 492 model of evolution, and 100 rapid bootstrap replicates (Data S5). Read coverages of these 493 metagenomic contigs were compared to measure relative abundances (Data S4).

\section{Chlamydiae species phylogeny}

495 Protein sequences from 74 single-copy marker genes (Data S7), found conserved across 496 chlamydiae MAGs and PVC species representatives (Data S6), were each aligned using MAFFT497 L-INS-i v7.471 (107), and trimmed with BMGE (108) (entropy of 0.6). IQTREE v. 1.6.12 (109) 498 was used to infer single-gene trees using ModelFinder (110) for model selection from LG (111) 499 and LG empirical mixture models (C10 to C60) (112), with gamma or free-distributed rates (+G or 
$500+\mathrm{R})(113)$, and with or without empirically determined amino acid frequencies $(+\mathrm{F})$. Trees were

501 manually inspected, divergent sequences removed, and the process repeated where necessary (Data

502 S7). To determine which marker genes had the strongest phylogenetic signal, the monophyly of

503 PVC phyla in the single-gene trees was assessed (Data S7). Four marker gene datasets were chosen

504 to include all genes (74 NOGs), genes where Chlamydiae was monophyletic (60 NOGs), genes

505 where Chlamydiae and most other PVC phyla were monophyletic (40 NOGs), and genes all PVC

506 phyla were monophyletic (15 NOGs) (Data S7). ML species phylogenies were then inferred as

507 described above for concatenated alignments of each dataset.

508 The topology across all species trees was consistent and the placement of sponge-associated

509 chlamydiae MAGs supported (Figure S3 and Data S5). Further analyses were thus performed with

510 only the smallest concatenated dataset (15 NOGs). A ML tree was inferred using IQTREE v. 1.6.12

511 (109) with 100 non-parametric bootstraps under the PMSF approximation (38) of the

$512 \mathrm{LG}+\mathrm{C} 60+\mathrm{F}+\mathrm{R} 4$ model of evolution (Figure 2a). A Bayesian phylogeny was also inferred using

513 PhyloBayes-MPI 1.7b (114) and the CAT $+\mathrm{GTR}+\Gamma 4$ model of evolution (115), with four

514 independent MCMC chains. After approximately 100,000 generations the four chains had not

515 converged (with a burn-in of 5000, and sampling every 10 generations) (Data S5). However, two

516 chains had converged (maxdiff of 0.16) (Figure 2a), and the topology of both deeper nodes and the

517 placement of sponge-associated chlamydiae was consistent across all chains (Data S5).

\section{Single-protein phylogenies of genes of interest}

519 The eukaryotic affiliation of typically-eukaryotic genes was confirmed using a phylogenetic 520 workflow (https://github.com/jennahd/HGT trees) (Data S5). Additionally, phylogenetic trees 521 were inferred for protein sequences with SnoaL-like PKS (PF07366) and PepM (PF13714) protein 522 domains (Data S5). Here, DIAMOND blastp (116) searches (with “max-target-seqs 2000” and

523 "more-sensitive") were performed against NCBI's NR database (117), and sequence redundancy 524 removed using CD-HIT v. 4.8.1 (118) at 80\% identity. For PepM, the top 100 hits to the Swiss525 Prot database, of proteins with curated annotations, were additionally retrieved (119). Sequences 526 were aligned and trimmed as above. For SnoaL-like PKS an initial tree was inferred using FastTree 5272 (120) and a subset of more distantly-related sequences removed. ML phylogenies was then 528 inferred using IQTREE v. 1.6.12 (109), with model selection by ModelFinder (110) as described 529 above. The $\mathrm{LG}+\mathrm{C} 60+\mathrm{F}+\mathrm{R} 4$ model was selected in both cases, and 1000 ultrafast bootstraps 530 inferred (121) (Data S5). 


\section{Small subunit rRNA gene phylogeny of sponge-associated Chlamydiae diversity}

532 Chlamydial SSU rRNA gene amplicon OTUs were collected from the present study, the prior study

533 of these marine sponge species (15), and from a survey of sponge microbial diversity (13). These

534 were combined with full-length and near full-length SSU rRNA genes from chlamydiae MAGs

535 and reference chlamydiae genomes (Data S6), and prior surveys of chlamydial (20). Sequences

536 were added to a previously published bacterial SSU rRNA gene alignment of $85 \%$ sequence

537 identity representatives (21) using MAFFT-L-INS-i v7.471 (107) (“--add” for near full-length

538 sequences, and “--addfragments" for amplicon OTUs). The alignment was trimmed using trimAl

539 v1.4.rev15 (122) with a gap threshold of 0.1. A ML phylogeny was then inferred using IQTREE v.

540 1.6.12 (109), with the GTR+F+R10 model selected from GTR models (123) by ModelFinder (110)

541 and 1000 ultrafast bootstraps (121).

\section{Environmental distribution}

543 The environmental distribution of specific chlamydial lineages was assessed using IMNGS (73).

544 Here, environmental samples were screened for sequences with at least 95\% identity to SSU rRNA

545 genes from several sponge-associated chlamydiae MAGs (Data S11). Samples with at least 0.1\%

546 relative abundance of these chlamydial lineages were also identified (Data S11).

\section{Data visualization and availability}

548 Phylogenetic trees were visualized using the ETE3 Toolkit (124), iTOL (125), and Figtree v1.4.4

549 (http://tree.bio.ed.ac.uk/software/figtree/). Plots were generated using R version 4.0.3 (R Core

550 Team, 2020), with ggplot2 v. 3.3.2 (126), and with UpSetR v. 1.4 .0 (127) for intersection plots.

551 Assembled metagenomes, metagenome sequence reads, amplicon sequence reads, and MAGs

552 generated from each sponge sample can be found deposited under BioProject PRJNA504765.

553 Accessions for data analyzed or obtained in this study can be found in Data S1, S4, and S6. Whole

554 Genome Shotgun projects for sponge metagenome assemblies P_S1, P_S2, P_S3, O_S4.1, O_S4.2,

555 O_S5, and X_S6 have been deposited at DDBJ/ENA/GenBank under the accessions

556 JAHZIM000000000, JAHZIN000000000， JAHZIO000000000， JAHZIP000000000,

557 JAHZIS000000000, JAHZIQ000000000, and JAHZIR000000000, respectively. The versions

558 described in this paper are versions JAHZIM010000000, JAHZIN010000000,

559 JAHZIO010000000, JAHZIP010000000, JAHZIS010000000, JAHZIQ010000000, and

560 JAHZIR010000000. Sequence alignments and phylogenetic trees in newick format are provided at

561 the Figshare data repository DOI: 10.6084/m9.figshare.14939475. 


\section{ACKNOWLEDGEMENTS}

562 We thank Klaske Schippers and Rene Wijffels for obtaining the sponge samples examined in this

563 study. We thank D. Tamarit for useful advice and discussions. Sequencing was performed at the

564 Uppsala Genome Centre, a sequencing platform that is hosted at Uppsala University as part of the

565 National Genomics Infrastructure at the Science for Life Laboratory. This national infrastructure

566 is supported by the Swedish Research Council (VR-RFI) and the Knut and Alice Wallenberg

567 Foundation. Computational resources were provided by the Swedish National Infrastructure for

568 Computing (SNIC) at UPPMAX with the project number SNIC 2020/15-158, PDC with the project

569 numbers SNIC 2019/3-474 and SNIC 2020/5-473, and HPC2N with the project number SNIC

570 2019/5-114. This work was supported by grants from the European Research Council (ERC

571 Starting and Consolidator grants 310039 and 817834, respectively), and the Swedish Research

572 Council (VR grant 2015-04959).

\section{COMPETING INTERESTS}

573 All authors declare that they have no competing financial interests in relation to the work described. 


\section{REFERENCES}

1. Hentschel U, Piel J, Degnan SM, Taylor MW. Genomic insights into the marine sponge microbiome. Nat Rev Microbiol. 2012;10(9):641-U75.

2. Reveillaud J, Maignien L, Eren AM, Huber JA, Apprill A, Sogin ML, et al. Host-specificity among abundant and rare taxa in the sponge microbiome. Isme J. 2014;8(6):1198-209.

3. Moitinho-Silva L, Nielsen S, Amir A, Gonzalez A, Ackermann GL, Cerrano C, et al. The sponge microbiome project. Gigascience. 2017;6(10):1-7.

4. Pita L, Rix L, Slaby BM, Franke A, Hentschel U. The sponge holobiont in a changing ocean: from microbes to ecosystems. Microbiome. 2018;6(1):46.

5. Paul VJ, Freeman CJ, Agarwal V. Chemical Ecology of Marine Sponges: New Opportunities through "Omics". Integr Comp Biol. 2019;59(4):765-76.

6. Taylor MW, Radax R, Steger D, Wagner M. Sponge-associated microorganisms: evolution, ecology, and biotechnological potential. Microbiol Mol Biol Rev. 2007;71(2):295-347.

7. Palazzotto E, Weber T. Omics and multi-omics approaches to study the biosynthesis of secondary metabolites in microorganisms. Curr Opin Microbiol. 2018;45:109-16.

8. Kenshole E, Herisse M, Michael M, Pidot SJ. Natural product discovery through microbial genome mining. Curr Opin Chem Biol. 2021;60:47-54.

9. Leal MC, Puga J, Serodio J, Gomes NCM, Calado R. Trends in the Discovery of New Marine Natural Products from Invertebrates over the Last Two Decades - Where and What Are We Bioprospecting? Plos One. 2012;7(1).

10. Egan S, Gardiner M. Microbial Dysbiosis: Rethinking Disease in Marine Ecosystems. Front Microbiol. 2016;7.

11. Schippers KJ, Sipkema D, Osinga R, Smidt H, Pomponi SA, Martens DE, et al. Cultivation of sponges, sponge cells and symbionts: achievements and future prospects. Adv Mar Biol. 2012;62:273-337.

12. Gutleben J, Loureiro C, Ramirez Romero LA, Shetty S, Wijffels RH, Smidt H, et al. Cultivation of Bacteria From Aplysina aerophoba: Effects of Oxygen and Nutrient Gradients. Front Microbiol. 2020;11:175.

13. Thomas T, Moitinho-Silva L, Lurgi M, Bjork JR, Easson C, Astudillo-Garcia C, et al. Diversity, structure and convergent evolution of the global sponge microbiome. Nat Commun. 2016;7:11870.

14. Zhu P, Li Q, Wang G. Unique microbial signatures of the alien Hawaiian marine sponge Suberites zeteki. Microb Ecol. 2008;55(3):406-14.

15. Naim MA, Morillo JA, Sorensen SJ, Waleed AA, Smidt H, Sipkema D. Host-specific microbial communities in three sympatric North Sea sponges. FEMS Microbiol Ecol. 2014;90(2):390-403.

16. Omsland A, Sixt BS, Horn M, Hackstadt T. Chlamydial metabolism revisited: interspecies metabolic variability and developmental stage-specific physiologic activities. Fems Microbiol Rev. 2014;38(4):779801.

17. Taylor-Brown A, Vaughan L, Greub G, Timms P, Polkinghorne A. Twenty years of research into Chlamydia-like organisms: a revolution in our understanding of the biology and pathogenicity of members of the phylum Chlamydiae. Pathog Dis. 2015;73(1).

18. Collingro A, Kostlbacher S, Horn M. Chlamydiae in the Environment. Trends Microbiol. 2020;28(11):87788.

19. Elwell C, Mirrashidi K, Engel J. Chlamydia cell biology and pathogenesis. Nat Rev Microbiol. 2016;14(6):385-400.

20. Lagkouvardos I, Weinmaier T, Lauro FM, Cavicchioli R, Rattei T, Horn M. Integrating metagenomic and amplicon databases to resolve the phylogenetic and ecological diversity of the Chlamydiae. Isme J. 2014;8(1):115-25.

21. Schulz F, Eloe-Fadrosh EA, Bowers RM, Jarett J, Nielsen T, Ivanova NN, et al. Towards a balanced view of the bacterial tree of life. Microbiome. 2017;5(1):140.

22. Dharamshi JE, Tamarit D, Eme L, Stairs CW, Martijn J, Homa F, et al. Marine Sediments Illuminate Chlamydiae Diversity and Evolution. Curr Biol. 2020;30(6):1032-48 e7.

23. Taylor-Brown A, Madden D, Polkinghorne A. Culture-independent approaches to chlamydial genomics. Microb Genomics. 2018;4(2).

24. Taylor-Brown A, Pillonel T, Greub G, Vaughan L, Nowak B, Polkinghorne A. Metagenomic Analysis of Fish-Associated $\mathrm{Ca}$. Parilichlamydiaceae Reveals Striking Metabolic Similarities to the Terrestrial Chlamydiaceae. Genome Biol Evol. 2018;10(10):2614-28. 
25. Taylor-Brown A, Spang L, Borel N, Polkinghorne A. Culture-independent metagenomics supports discovery of uncultivable bacteria within the genus Chlamydia. Sci Rep-Uk. 2017;7.

26. Köstlbacher S, Collingro A, Halter T, Schulz F, Jungbluth SP, Horn M. Pangenomics reveals alternative environmental lifestyles among chlamydiae. Nature Communications. 2021;12:4021.

27. Collingro A, Kostlbacher S, Mussmann M, Stepanauskas R, Hallam SJ, Horn M. Unexpected genomic features in widespread intracellular bacteria: evidence for motility of marine chlamydiae. Isme J. 2017;11(10):2334-44.

28. Wagner M, Horn M. The Planctomycetes, Verrucomicrobia, Chlamydiae and sister phyla comprise a superphylum with biotechnological and medical relevance. Curr Opin Biotechnol. 2006;17(3):241-9.

29. Devos DP, Ward NL. Mind the PVCs. Environ Microbiol. 2014;16(5):1217-21.

30. Wichels A, Wurtz S, Dopke H, Schutt C, Gerdts G. Bacterial diversity in the breadcrumb sponge Halichondria panicea (Pallas). FEMS Microbiol Ecol. 2006;56(1):102-18.

31. Bayer K, Busch K, Kenchington E, Beazley L, Franzenburg S, Michels J, et al. Microbial Strategies for Survival in the Glass Sponge Vazella pourtalesii. mSystems. 2020;5(4).

32. Israelsson O. Chlamydial symbionts in the enigmatic Xenoturbella (Deuterostomia). J Invertebr Pathol. 2007;96(3):213-20.

33. Pizzetti I, Schulz F, Tyml T, Fuchs BM, Amann R, Horn M, et al. Chlamydial seasonal dynamics and isolation of 'Candidatus Neptunochlamydia vexilliferae' from a Tyrrhenian coastal lake. Environ Microbiol. 2016;18(8):2405-17.

34. Anantharaman K, Brown CT, Hug LA, Sharon I, Castelle CJ, Probst AJ, et al. Thousands of microbial genomes shed light on interconnected biogeochemical processes in an aquifer system. Nature Communications. 2016;7.

35. Karlsen M, Nylund A, Watanabe K, Helvik JV, Nylund S, Plarre H. Characterization of 'Candidatus Clavochlamydia salmonicola': an intracellular bacterium infecting salmonid fish. Environ Microbiol. 2008;10(1):208-18.

36. Nunes A, Gomes JP. Evolution, phylogeny, and molecular epidemiology of Chlamydia. Infect Genet Evol. 2014;23:49-64.

37. Huerta-Cepas J, Szklarczyk D, Forslund K, Cook H, Heller D, Walter MC, et al. eggNOG 4.5: a hierarchical orthology framework with improved functional annotations for eukaryotic, prokaryotic and viral sequences. Nucleic Acids Res. 2016;44(D1):D286-93.

38. Wang HC, Minh BQ, Susko E, Roger AJ. Modeling Site Heterogeneity with Posterior Mean Site Frequency Profiles Accelerates Accurate Phylogenomic Estimation. Syst Biol. 2018;67(2):216-35.

39. Gauthier MEA, Watson JR, Degnan SM. Draft Genomes Shed Light on the Dual Bacterial Symbiosis that Dominates the Microbiome of the Coral Reef Sponge Amphimedon queenslandica. Front Mar Sci. 2016;3.

40. Engelberts JP, Robbins SJ, de Goeij JM, Aranda M, Bell SC, Webster NS. Characterization of a sponge microbiome using an integrative genome-centric approach. Isme J. 2020;14(5):1100-10.

41. Clifford EL, Hansell DA, Varela MM, Nieto-Cid M, Herndl GJ, Sintes E. Crustacean zooplankton release copious amounts of dissolved organic matter as taurine in the ocean. Limnol Oceanogr. 2017;62(6):274558.

42. Fieseler L, Horn M, Wagner M, Hentschel U. Discovery of the novel candidate phylum "Poribacteria" in marine sponges. Appl Environ Microb. 2004;70(6):3724-32.

43. Kamke J, Sczyrba A, Ivanova N, Schwientek P, Rinke C, Mavromatis K, et al. Single-cell genomics reveals complex carbohydrate degradation patterns in poribacterial symbionts of marine sponges. Isme J. 2013;7(12):2287-300.

44. Podell S, Blanton JM, Neu A, Agarwal V, Biggs JS, Moore BS, et al. Pangenomic comparison of globally distributed Poribacteria associated with sponge hosts and marine particles. Isme J. 2019;13(2):468-81.

45. Michell RH. Inositol derivatives: evolution and functions. Nat Rev Mol Cell Biol. 2008;9(2):151-61.

46. Gilk SD, Beare PA, Heinzen RA. Coxiella burnetii expresses a functional Delta24 sterol reductase. J Bacteriol. 2010;192(23):6154-9.

47. Meadows JA, Wargo MJ. Carnitine in bacterial physiology and metabolism. Microbiol-Sgm. 2015;161:1161-74.

48. Slaby BM, Hackl T, Horn H, Bayer K, Hentschel U. Metagenomic binning of a marine sponge microbiome reveals unity in defense but metabolic specialization. Isme J. 2017;11(11):2465-78.

49. Xiao ZJ, Xu P. Acetoin metabolism in bacteria. Crit Rev Microbiol. 2007;33(2):127-40.

50. Yang T, Rao Z, Zhang X, Xu M, Xu Z, Yang ST. Metabolic engineering strategies for acetoin and 2,3butanediol production: advances and prospects. Crit Rev Biotechnol. 2017;37(8):990-1005. 
51. Xiao Z, Lu JR. Strategies for enhancing fermentative production of acetoin: a review. Biotechnol Adv. 2014;32(2):492-503.

52. Chistoserdova L. Modularity of methylotrophy, revisited. Environ Microbiol. 2011;13(10):2603-22.

53. Moitinho-Silva L, Seridi L, Ryu T, Voolstra CR, Ravasi T, Hentschel U. Revealing microbial functional activities in the Red Sea sponge Stylissa carteri by metatranscriptomics. Environ Microbiol. 2014;16(12):3683-98.

54. Ufarte L, Laville E, Duquesne S, Potocki-Veronese G. Metagenomics for the discovery of pollutant degrading enzymes. Biotechnol Adv. 2015;33(8):1845-54.

55. Podell S, Blanton JM, Oliver A, Schorn MA, Agarwal V, Biggs JS, et al. A genomic view of trophic and metabolic diversity in clade-specific Lamellodysidea sponge microbiomes. Microbiome. 2020;8(1):97.

56. Moiseeva OV, Solyanikova IP, Kaschabek SR, Groning J, Thiel M, Golovleva LA, et al. A new modified ortho cleavage pathway of 3-chlorocatechol degradation by Rhodococcus opacus 1CP: genetic and biochemical evidence. J Bacteriol. 2002;184(19):5282-92.

57. Arora PK, Bae H. Bacterial degradation of chlorophenols and their derivatives. Microb Cell Fact. 2014;13(1):31.

58. Rust M, Helfrich EJN, Freeman MF, Nanudorn P, Field CM, Ruckert C, et al. A multiproducer microbiome generates chemical diversity in the marine sponge Mycale hentscheli. P Natl Acad Sci USA. 2020;117(17):9508-18.

59. Medema MH, Blin K, Cimermancic P, de Jager V, Zakrzewski P, Fischbach MA, et al. antiSMASH: rapid identification, annotation and analysis of secondary metabolite biosynthesis gene clusters in bacterial and fungal genome sequences. Nucleic Acids Res. 2011;39:W339-W46.

60. Falagas ME, Athanasaki F, Voulgaris GL, Triarides NA, Vardakas KZ. Resistance to fosfomycin: Mechanisms, Frequency and Clinical Consequences. Int J Antimicrob Agents. 2019;53(1):22-8.

61. Metcalf WW, van der Donk WA. Biosynthesis of phosphonic and phosphinic acid natural products. Annu Rev Biochem. 2009; 78:65-94.

62. McCoy AJ, Sandlin RC, Maurelli AT. In vitro and in vivo functional activity of Chlamydia MurA, a UDP$\mathrm{N}$-acetylglucosamine enolpyruvyl transferase involved in peptidoglycan synthesis and fosfomycin resistance. J Bacteriol. 2003;185(4):1218-28.

63. Graca AP, Calisto R, Lage OM. Planctomycetes as Novel Source of Bioactive Molecules. Front Microbiol. $2016 ; 7$.

64. Wiegand S, Jogler M, Jogler C. On the maverick Planctomycetes. Fems Microbiol Rev. 2018;42(6):739-60.

65. O'Brien J, Wright GD. An ecological perspective of microbial secondary metabolism. Curr Opin Biotech. 2011;22(4):552-8.

66. Tianero MD, Balaich JN, Donia MS. Localized production of defence chemicals by intracellular symbionts of Haliclona sponges. Nat Microbiol. 2019;4(7):1149-59.

67. Maita C, Matsushita M, Miyoshi M, Okubo T, Nakamura S, Matsuo J, et al. Amoebal endosymbiont Neochlamydia protects host amoebae against Legionella pneumophila infection by preventing Legionella entry. Microbes Infect. 2018;20(4):236-44.

68. Konig L, Wentrup C, Schulz F, Wascher F, Escola S, Swanson MS, et al. Symbiont-Mediated Defense against Legionella pneumophila in Amoebae. mBio. 2019;10(3).

69. Abdelmohsen UR, Cheng C, Reimer A, Kozjak-Pavlovic V, Ibrahim AK, Rudel T, et al. Antichlamydial Sterol from the Red Sea Sponge Callyspongia aff. implexa. Planta Med. 2015;81(5):382-7.

70. Gaikwad S, Shouche YS, Gade WN. Microbial community structure of two freshwater sponges using Illumina MiSeq sequencing revealed high microbial diversity. Amb Express. 2016;6.

71. Angly FE, Pantos O, Morgan TC, Rich V, Tonin H, Bourne DG, et al. Diuron tolerance and potential degradation by pelagic microbiomes in the Great Barrier Reef lagoon. PeerJ. 2016;4:e1758.

72. Knobloch S, Johannsson R, Marteinsson V. Bacterial diversity in the marine sponge Halichondria panicea from Icelandic waters and host-specificity of its dominant symbiont "Candidatus Halichondribacter symbioticus". FEMS Microbiol Ecol. 2019;95(1).

73. Lagkouvardos I, Joseph D, Kapfhammer M, Giritli S, Horn M, Haller D, et al. IMNGS: A comprehensive open resource of processed 16S rRNA microbial profiles for ecology and diversity studies. Sci Rep. 2016;6:33721.

74. Klindworth A, Pruesse E, Schweer T, Peplies J, Quast C, Horn M, et al. Evaluation of general 16S ribosomal RNA gene PCR primers for classical and next-generation sequencing-based diversity studies. Nucleic Acids Res. 2013;41(1):e1. 
75. Martin M. Cutadapt removes adapter sequences from high-throughput sequencing reads. EMBnetJournal. 2011;17(1):10-2.

76. Rognes T, Flouri T, Nichols B, Quince C, Mahe F. VSEARCH: a versatile open source tool for metagenomics. PeerJ. 2016;4:e2584.

77. Edgar RC, Haas BJ, Clemente JC, Quince C, Knight R. UCHIME improves sensitivity and speed of chimera detection. Bioinformatics. 2011;27(16):2194-200.

78. Quast C, Pruesse E, Yilmaz P, Gerken J, Schweer T, Yarza P, et al. The SILVA ribosomal RNA gene database project: improved data processing and web-based tools. Nucleic Acids Res. 2013;41(Database issue):D590-6.

79. Lanzen A, Jorgensen SL, Huson DH, Gorfer M, Grindhaug SH, Jonassen I, et al. CREST--classification resources for environmental sequence tags. PLoS One. 2012;7(11):e49334.

80. Bolger AM, Lohse M, Usadel B. Trimmomatic: a flexible trimmer for Illumina sequence data. Bioinformatics. 2014;30(15):2114-20.

81. Andrews S. FastQC: a quality control tool for high throughput sequence data. http://www.bioinformatics.babraham.ac.uk/projects/fastqc2010.

82. Li D, Liu CM, Luo R, Sadakane K, Lam TW. MEGAHIT: an ultra-fast single-node solution for large and complex metagenomics assembly via succinct de Bruijn graph. Bioinformatics. 2015;31(10):1674-6.

83. Gurevich A, Saveliev V, Vyahhi N, Tesler G. QUAST: quality assessment tool for genome assemblies. Bioinformatics. 2013;29(8):1072-5.

84. Hyatt D, Chen GL, LoCascio PF, Land ML, Larimer FW, Hauser LJ. Prodigal: prokaryotic gene recognition and translation initiation site identification. Bmc Bioinformatics. 2010;11.

85. Seemann T. barrnap 0.8: rapid ribosomal RNA prediction. https://github.com/tseemann/barrnap2013.

86. Langmead B, Salzberg SL. Fast gapped-read alignment with Bowtie 2. Nat Methods. 2012;9(4):357-9.

87. Kang DD, Li F, Kirton E, Thomas A, Egan R, An H, et al. MetaBAT 2: an adaptive binning algorithm for robust and efficient genome reconstruction from metagenome assemblies. PeerJ. 2019;7:e7359.

88. Alneberg J, Bjarnason BS, de Bruijn I, Schirmer M, Quick J, Ijaz UZ, et al. Binning metagenomic contigs by coverage and composition. Nat Methods. 2014;11(11):1144-6.

89. Wu YW, Simmons BA, Singer SW. MaxBin 2.0: an automated binning algorithm to recover genomes from multiple metagenomic datasets. Bioinformatics. 2016;32(4):605-7.

90. Uritskiy GV, DiRuggiero J, Taylor J. MetaWRAP-a flexible pipeline for genome-resolved metagenomic data analysis. Microbiome. 2018;6(1):158.

91. Eren AM, Kiefl E, Shaiber A, Veseli I, Miller SE, Schechter MS, et al. Community-led, integrated, reproducible multi-omics with anvi'o. Nat Microbiol. 2021;6(1):3-6.

92. Eren AM, Esen OC, Quince C, Vineis JH, Morrison HG, Sogin ML, et al. Anvi'o: an advanced analysis and visualization platform for 'omics data. PeerJ. 2015;3:e1319.

93. Seemann T. Prokka: rapid prokaryotic genome annotation. Bioinformatics. 2014;30(14):2068-9.

94. Camacho C, Coulouris G, Avagyan V, Ma N, Papadopoulos J, Bealer K, et al. BLAST+: architecture and applications. Bmc Bioinformatics. 2009;10:421.

95. El-Gebali S, Mistry J, Bateman A, Eddy SR, Luciani A, Potter SC, et al. The Pfam protein families database in 2019. Nucleic Acids Res. 2019;47(D1):D427-D32.

96. Haft DH, Selengut JD, White O. The TIGRFAMs database of protein families. Nucleic Acids Res. 2003;31(1):371-3.

97. Jones P, Binns D, Chang HY, Fraser M, Li W, McAnulla C, et al. InterProScan 5: genome-scale protein function classification. Bioinformatics. 2014;30(9):1236-40.

98. Hugoson E, Lam WT, Guy L. miComplete: weighted quality evaluation of assembled microbial genomes. Bioinformatics. 2020;36(3):936-7.

99. Huerta-Cepas J, Forslund K, Coelho LP, Szklarczyk D, Jensen LJ, von Mering C, et al. Fast Genome-Wide Functional Annotation through Orthology Assignment by eggNOG-Mapper. Molecular Biology and Evolution. 2017;34(8):2115-22.

100. Kanehisa M, Sato Y, Kawashima M, Furumichi M, Tanabe M. KEGG as a reference resource for gene and protein annotation. Nucleic Acids Res. 2016;44(D1):D457-62.

101. Kanehisa M, Sato Y, Morishima K. BlastKOALA and GhostKOALA: KEGG Tools for Functional Characterization of Genome and Metagenome Sequences. J Mol Biol. 2016;428(4):726-31.

102. Galperin MY, Wolf YI, Makarova KS, Vera Alvarez R, Landsman D, Koonin EV. COG database update: focus on microbial diversity, model organisms, and widespread pathogens. Nucleic Acids Res.

2021;49(D1):D274-D81. 
103. Kautsar SA, Blin K, Shaw S, Navarro-Munoz JC, Terlouw BR, van der Hooft JJJ, et al. MIBiG 2.0: a repository for biosynthetic gene clusters of known function. Nucleic Acids Res. 2020;48(D1):D454-D8.

104. Nomura M, Morgan EA. Genetics of bacterial ribosomes. Annu Rev Genet. 1977;11:297-347.

105. Martijn J, Vosseberg J, Guy L, Offre P, Ettema TJG. Deep mitochondrial origin outside the sampled alphaproteobacteria. Nature. 2018;557(7703):101-5.

106. Stamatakis A. RAxML version 8: a tool for phylogenetic analysis and post-analysis of large phylogenies. Bioinformatics. 2014;30(9):1312-3.

107. Katoh K, Standley DM. MAFFT multiple sequence alignment software version 7: improvements in performance and usability. Mol Biol Evol. 2013;30(4):772-80.

108. Criscuolo A, Gribaldo S. BMGE (Block Mapping and Gathering with Entropy): a new software for selection of phylogenetic informative regions from multiple sequence alignments. BMC Evol Biol. 2010;10:210.

109. Nguyen LT, Schmidt HA, von Haeseler A, Minh BQ. IQ-TREE: a fast and effective stochastic algorithm $\mathrm{f}$ or estimating maximum-likelihood phylogenies. Mol Biol Evol. 2015;32(1):268-74.

110. Kalyaanamoorthy S, Minh BQ, Wong TKF, von Haeseler A, Jermiin LS. ModelFinder: fast model selection for accurate phylogenetic estimates. Nat Methods. 2017;14(6):587-9.

111. Le SQ, Gascuel O. An improved general amino acid replacement matrix. Mol Biol Evol. 2008;25(7):130720.

112. Quang le S, Gascuel O, Lartillot N. Empirical profile mixture models for phylogenetic reconstruction. Bioinformatics. 2008;24(20):2317-23.

113. Le SQ, Dang CC, Gascuel O. Modeling Protein Evolution with Several Amino Acid Replacement Matrices Depending on Site Rates. Molecular Biology and Evolution. 2012;29(10):2921-36.

114. Lartillot N, Rodrigue N, Stubbs D, Richer J. PhyloBayes MPI: phylogenetic reconstruction with infinite mixtures of profiles in a parallel environment. Syst Biol. 2013;62(4):611-5.

115. Lartillot N, Philippe H. A Bayesian mixture model for across-site heterogeneities in the amino-acid replacement process. Mol Biol Evol. 2004;21(6):1095-109.

116. Buchfink B, Xie C, Huson DH. Fast and sensitive protein alignment using DIAMOND. Nature Methods. 2015;12(1):59-60.

117. Sayers EW, Beck J, Bolton EE, Bourexis D, Brister JR, Canese K, et al. Database resources of the National Center for Biotechnology Information. Nucleic Acids Res. 2021;49(D1):D10-D7.

118. Li W, Godzik A. Cd-hit: a fast program for clustering and comparing large sets of protein or nucleotide sequences. Bioinformatics. 2006;22(13):1658-9.

119. UniProt C. UniProt: the universal protein knowledgebase in 2021. Nucleic Acids Res. 2021;49(D1):D480D9.

120. Price MN, Dehal PS, Arkin AP. FastTree 2--approximately maximum-likelihood trees for large alignments. PLoS One. 2010;5(3):e9490.

121. Minh BQ, Nguyen MA, von Haeseler A. Ultrafast approximation for phylogenetic bootstrap. Mol Biol Evol. 2013;30(5):1188-95.

122. Capella-Gutierrez S, Silla-Martinez JM, Gabaldon T. trimAl: a tool for automated alignment trimming in large-scale phylogenetic analyses. Bioinformatics. 2009;25(15):1972-3.

123. Tavaré S. Some probabilistic and statistical problems in the analysis of DNA sequences. Lect Math Life Sci. 1986; 17:57-86.

124. Huerta-Cepas J, Serra F, Bork P. ETE 3: Reconstruction, Analysis, and Visualization of Phylogenomic Data. Mol Biol Evol. 2016;33(6):1635-8.

125. Letunic I, Bork P. Interactive tree of life (iTOL) v3: an online tool for the display and annotation of phylogenetic and other trees. Nucleic Acids Res. 2016;44(W1):W242-5.

126. Wickham H. ggplot2: Elegant Graphics for Data Analysis. Springer-Verlag New York. 2016.

127. Conway JR, Lex A, Gehlenborg N. UpSetR: an R package for the visualization of intersecting sets and their properties. Bioinformatics. 2017;33(18):2938-40. 


\section{Sample collection and preparation}

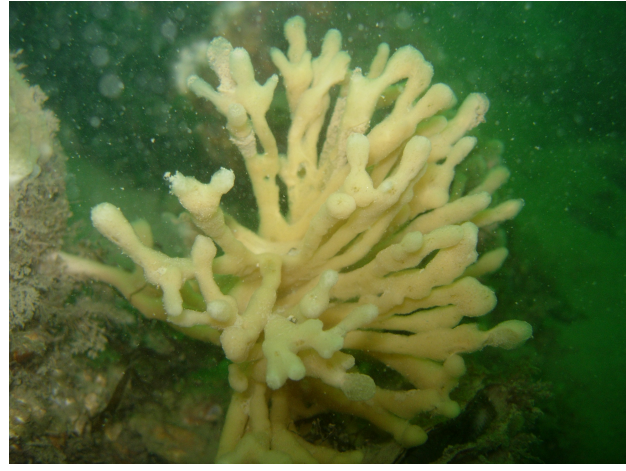

Sponge samples collected from the Oosterchelde estuary, Netherlands. (Haliclona oculata in image)

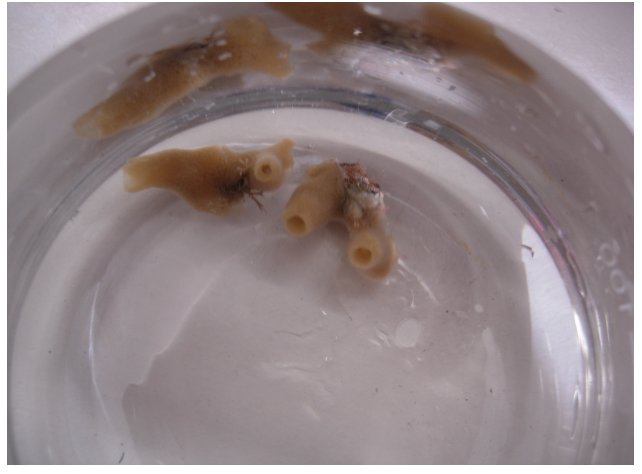

Samples washed in autoclaved seawater then stored at $-80^{\circ} \mathrm{C}$.

(Haliclona xena in image)

\begin{tabular}{|llll}
\multicolumn{1}{ll}{ ID } & Species & Sampling date and location \\
\hline P_S1 & Halichondria panicea & 2008-12-03: Oosterschelde/East \\
P_S2 & Halichondria panicea & 2008-12-03: Oosterschelde/East \\
P_S3 & Halichondria panicea & 2009-11-17: Oosterschelde \\
O_S4 & Haliclona oculata & 2008-12-03: Oosterschelde/East \\
O_S5 & Haliclona oculata & 2009-02-03: Lokkersnol \\
X_S6 & Haliclona xena & 2009-02-03: Lokkersnol
\end{tabular}

\section{DNA extraction}

Sponge associated DNA

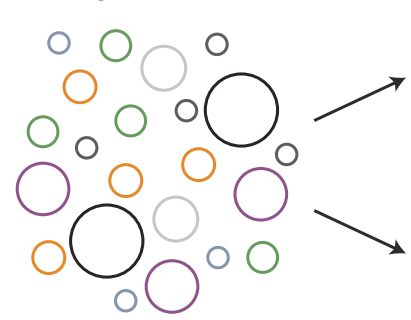

PowerLyser Powersoil DNA Extraction (P_S1, P_S2, P_S3, O_S4, O_S5, X_S6)

High Molecular Weight DNA Extraction (P_S1, P_S2, P_S3, O_S4)
SSU rRNA gene amplicons

Metagenome sequencing (O_S4.2, O_S5, X_S6)

Metagenome sequencing (P_S1, P_S2, P_S3, O_S4.1)

\section{Obtaining metagenome-assembled genomes}

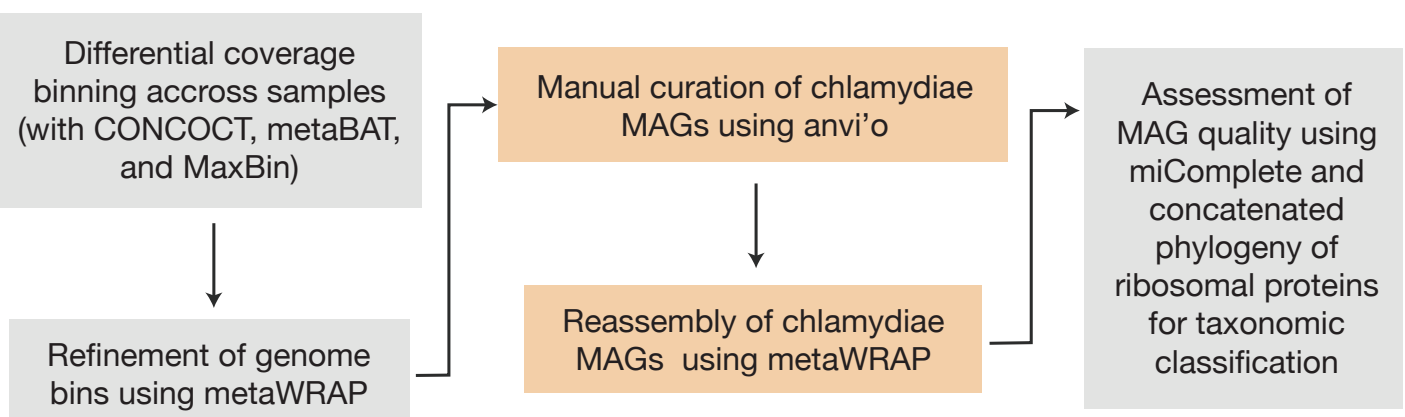

Figure S1. Methods overview of sample collection, sponge specimens collected, DNA extraction methods, amplicon sequencing, metagenome sequencing and assembly, and MAG binning and refinement. 
bioRxiv preprint doi: https://doi.org/10.1101/2021.12.21.473556; this version posted December 22, 2021. The copyright holder for this preprint (which was not certified by peer review) is the author/funder, who has granted bioRxiv a license to display the preprint in perpetuity. It is made available under aCC-BY-NC-ND 4.0 International license.

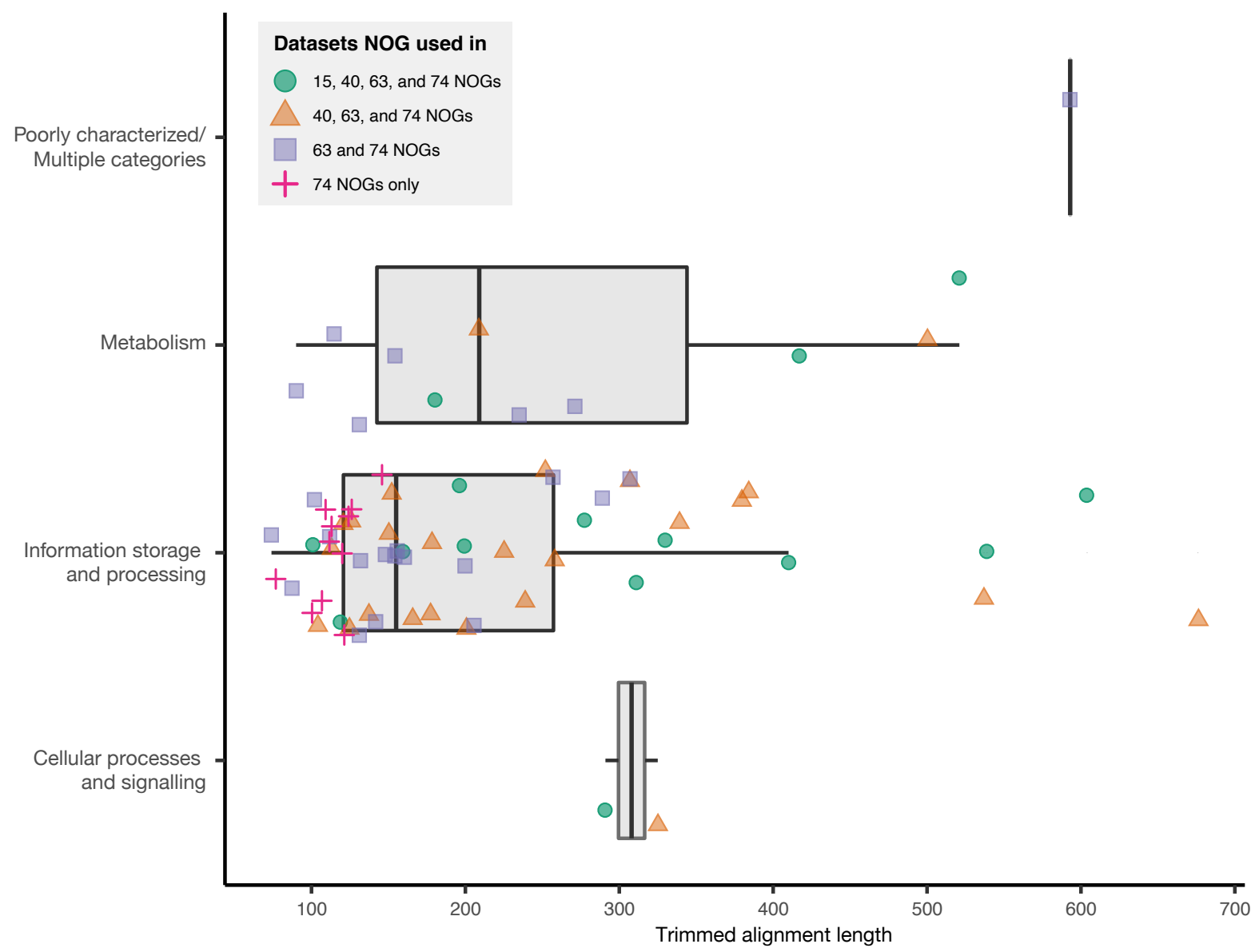

Figure S2. COG category membership of the 74 marker gene NOGs included in Chlamydiae species tree concatenated datasets alongside their trimmed alignment lengths. NOGs were assigned to datasets based on phylogenetic signal, measured by resolving Chlamydiae and other PVC phyla as monophyletic in single-protein trees. All marker gene NOGs were included in the 74 NOG dataset, those resolving Chlamydiae as monophyletic in the 63 NOG dataset, those resolving Chlamydiae and most PVC phyla as monophyletic in the 40 NOG dataset, and those resolving all PVC phyla as monophyletic in the 15 NOG dataset. See Data S7 for tree refinement and selection details. 
bioRxiv preprint doi: https://doi.org/10.1101/2021.12.21.473556; this version posted December 22, 2021. The copyright holder for this preprint (which was not certified by peer review) is the author/funder, who has granted bioRxiv a license to display the preprint in perpetuity. It is made available under aCC-BY-NC-ND 4.0 International license.

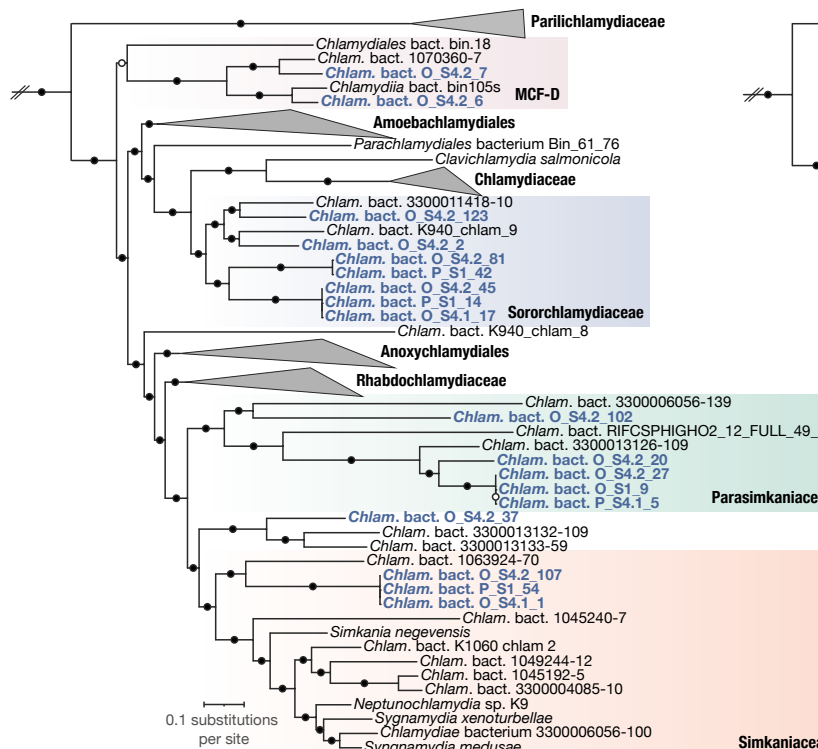

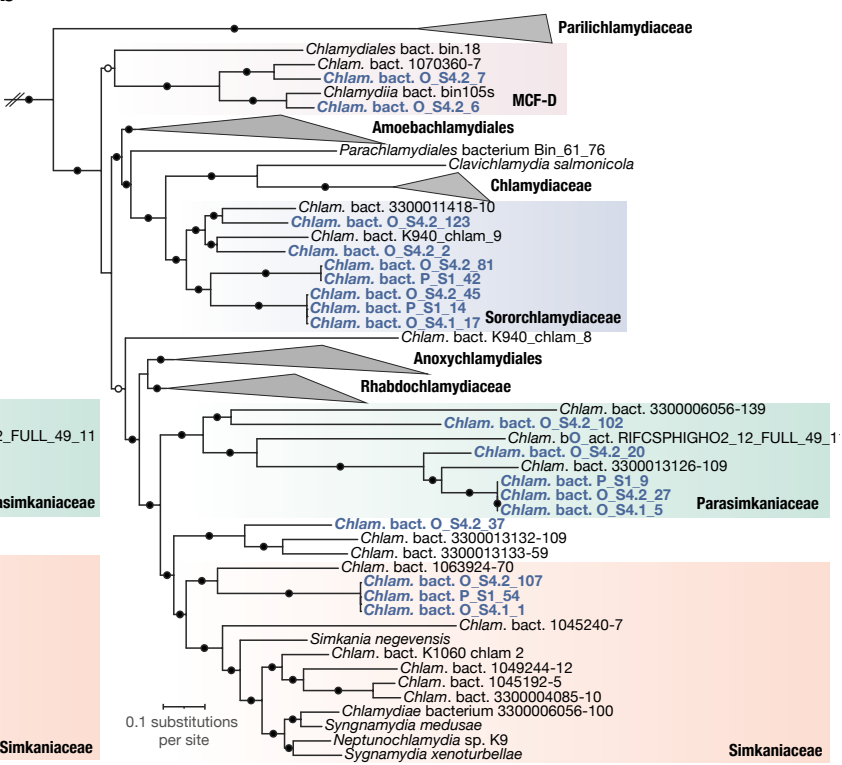

d

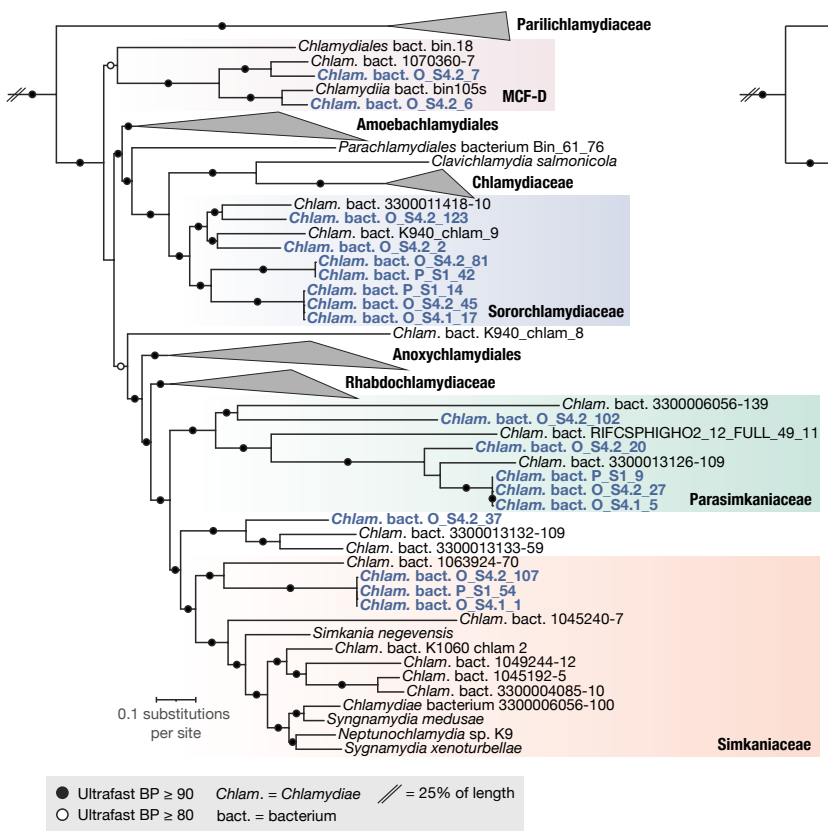

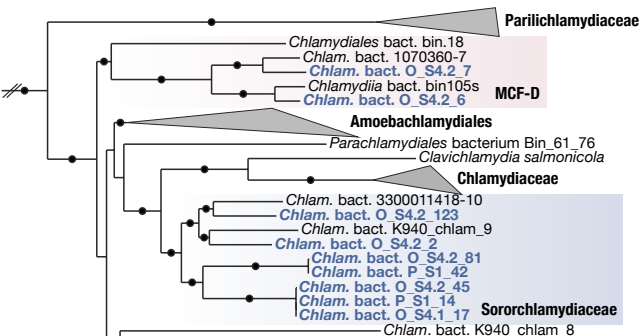

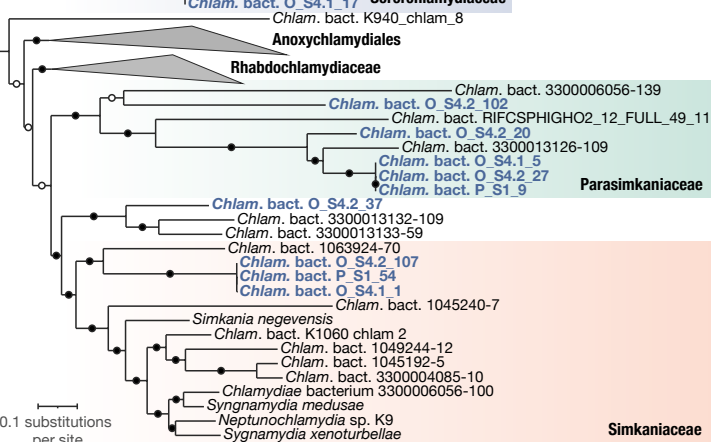

per site

Figure S3. The topology of Chlamydiae species relationships is consistent across reconstructions using subsets of the larger initial dataset of 74 NOGs. Concatenated maximum-likelihood protein phylogenies of Chlamydiae species inferred under the LG+C60+F+R4 model of evolution with 74 (a), 63 (b), 40 (c), and 15 (d) single-copy marker NOGs with 16760, 15502, 11211, and 4757 amino acid alignment positions, respectively. Trees are rooted by a PVC outgroup (not shown), with this branch reduced $25 \%$ in length. Sponge chlamydiae MAGs are coloured in blue, with relevant families coloured, and phylogroups with high relative abundance highlighted. Branch support is indicated by circles according to the legend. See Data S5 for uncollapsed species trees and Data S6 for NOGs included in each phylogenetic dataset. 
bioRxiv preprint doi: https://doi.org/10.1101/2021.12.21.473556; this version posted December 22, 2021. The copyright holder for this preprint (which was not certified by peer review) is the author/funder, who has granted bioRxiv a license to display the preprint in perpetuity. It is made available under aCC-BY-NC-ND 4.0 International license.

a
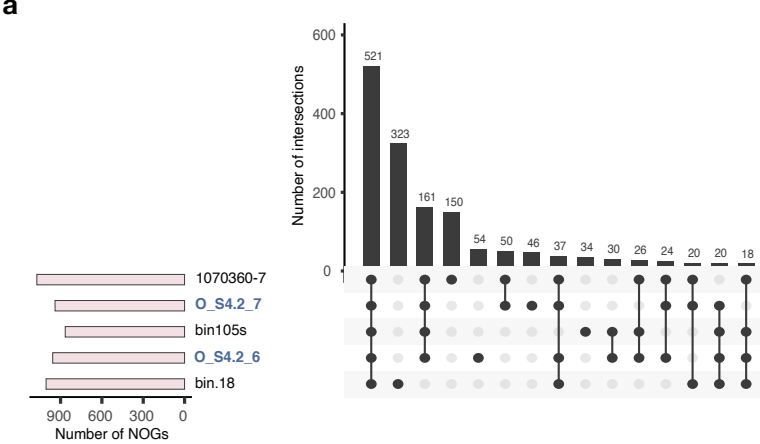

C

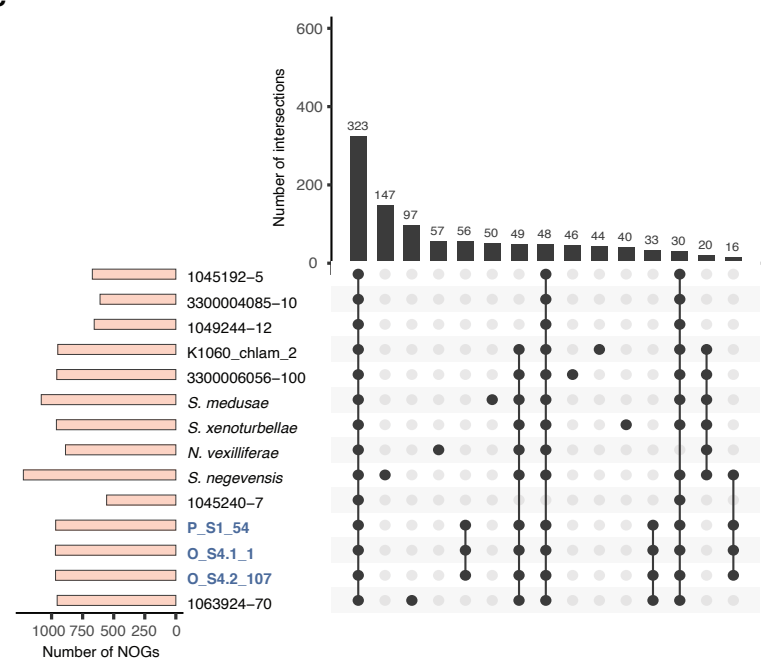

b
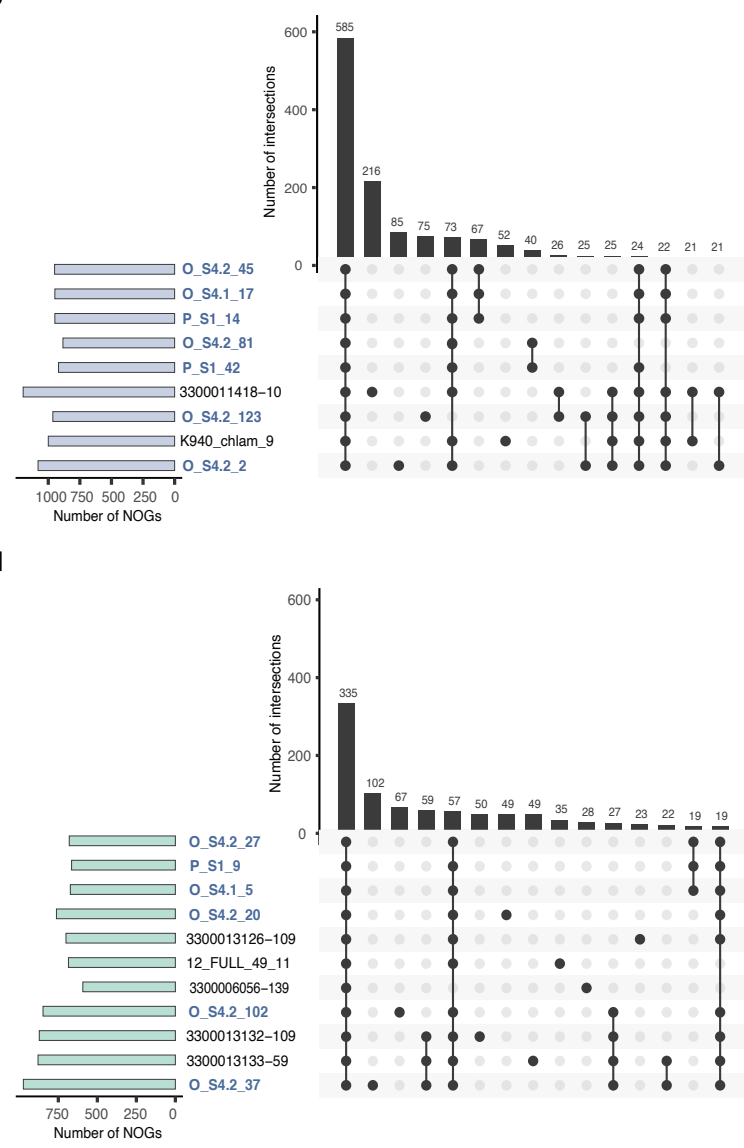

Figure S4. Chlamydiae families share varying levels of core gene content, with smaller sets of genes specific to sub-groups of sponge MAGs. Intersection plots give an overview of shared gene content across chlamydiae genomes from the families MCF-D (a), Ca. Sororchlamydiaceae (b), Ca. Parasimkaniaceae (c), and Simkaniaceae (d). Intersections are based on sets of NOGs found in each genome, with the total number of identified NOGs found in bar charts to the left of each taxon. Each intersection plot shows the number of NOGs shared between the taxa indicated by black circles for the top 15 most abundant intersections. 
bioRxiv preprint doi: https://doi.org/10.1101/2021.12.21.473556; this version posted December 22, 2021. The copyright holder for this preprint (which was not certified by peer review) is the author/funder, who has granted bioRxiv a license to display the preprint in perpetuity. It is made

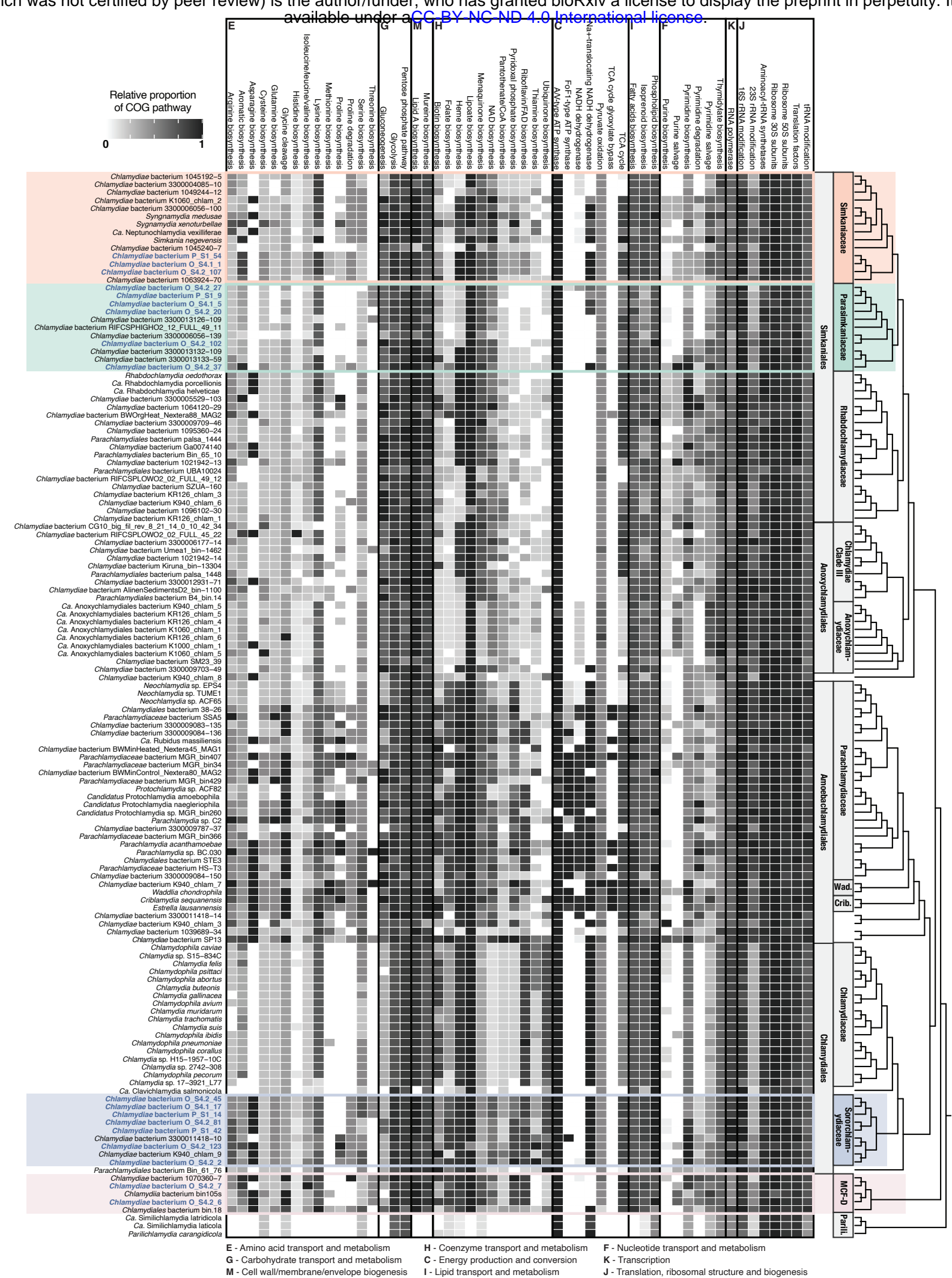

Figure S5. Overview of clusters of orthologous group (COG) pathways across Chlamydiae indicate that spongeassociated chlamydiae have similar metabolic profiles to other chlamydiae from their respective families. The heatmap is organized by COG category and shows the proportion of COGs from a pathway found across taxa, relative to the maximum among chlamydiae. Sponge-associated chlamydiae MAGs are in blue, with relevant families coloured accordingly. Order and family names are indicated to the right alongside a cladogram of species relationships, with the following short forms: Criblamydiaceae (Crib.), Parilichlamydiaceae (Parili.), and Waddliaceae (Wad.). See Data S9 for an overview of each COG across Chlamydiae genomes. 
bioRxiv preprint doi: https://doi.org/10.1101/2021.12.21.473556; this version posted December 22, 2021. The copyright holder for this preprint (which was not certified by peer review) is the author/funder, who has granted bioRxiv a license to display the preprint in perpetuity. It is made available under aCC-BY-NC-ND 4.0 International license.

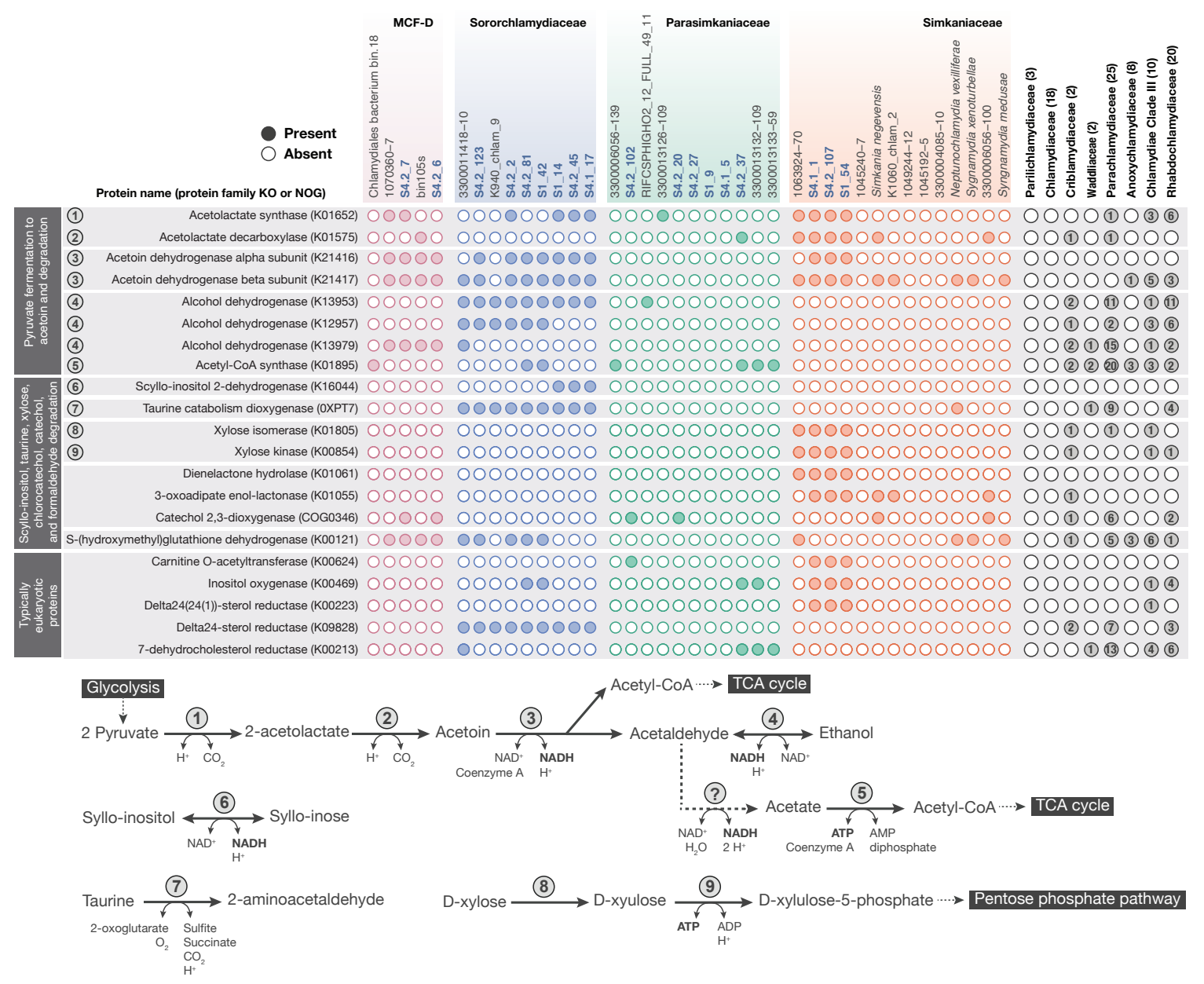

Figure S6. Sponge-associated chlamydiae genomes are enriched in genes related to fermentation, degradation, and genes typical of eukaryotes. The presence and absence of genes of interest across chlamydiae genomes from families with sponge-associated members is shown, alongside a schematic overview of pyruvate to acetoin fermentation, and the degradation of acetoin, syllo-inositol, taurine, and D-xylose. Gene function and protein domain are indicated to the left alongside numbers corresponding to the schematic overview. The number of representative genomes from other chlamydial families encoding the given protein family is indicated to the right. See Data S8 for a full overview of presence and absence across chlamydiae representatives and corresponding gene annotations for sponge-associated chlamydiae. 
bioRxiv preprint doi: https://doi.org/10.1101/2021.12.21.473556; this version posted December 22, 2021. The copyright holder for this preprint (which was not certified by peer review) is the author/funder, who has granted bioRxiv a license to display the preprint in perpetuity. It is made SUPPLEMENTARY DA Typilable under aCC-BY-NC-ND 4.0 International license.

Data S1. Sponge sample metadata and data accessions, metagenome assembly statistics, and confirmation of sponge identity based on metagenomic SSU rRNA genes.

Data S2. Bacteria-specific SSU rRNA gene amplicon OTU sequences.

Data S3. Overview of phylum and OTU relative abundances, read counts, and taxonomy for bacteria-specific SSU rRNA gene amplicons across sponge samples.

Data S4. Sponge metagenome microbial diversity and MAG information. Contigs encoding SSU rRNA genes and ribosomal proteins identified in metagenomes. Overview of MAGs retrieved from each metagenome, including bin statistics and taxoomic classification.

Data S5. Uncollapsed phylogenetic trees, including ribosomal protein phylogeny of metagenomic contigs, species phylogenies of concatenated marker proteins, single protein phylogenies including phosphoenolpyruvate mutase and SnoaL-like polyketide cyclase, and the SSU rRNA gene phylogeny.

Data S6. Summary of genome characteristics, IDs, and accessions of sponge chlamydiae MAGs and PVC species representatives.

Data S7. Single-copy marker proteins used in concatenated species phylogenies, sequences removed during refinement, and monophyly of PVC phyla in each single-protein tree.

Data S8. Annotations of proteins of interest, and the number of respective NOGs and KEGG KOs across chlamydiae.

Data S9. Overview of the presence and absence of COGs in each COG pathway across chlamydiae, and annotations of the corresponding proteins from sponge chlamydiae MAGs.

Data S10. Overview of antiSMASH results with the type and number of BGCs identified in each chlamydiae species representative. BGCs with top MiBIG hits are indicated alongside top hit information.

Data S11. Prevalence of close relatives of sponge chlamydiae (95\% identity) across environmental samples measured by IMNGS and based on SRA SSU rRNA gene amplicon datasets. SRA samples identified with at least $0.1 \%$ relative abundance. 